DESY 06-043

hep-ph/0604160

April 2006

\title{
QCD Corrections to Semi-Inclusive Hadron Production in Electron-Positron Annihilation at Two Loops
}

\author{
A. Mitov and S. Moch \\ Deutsches Elektronensynchrotron DESY \\ Platanenallee 6, D-15738 Zeuthen, Germany
}

\begin{abstract}
We report on the analytic calculation of the second-order QCD corrections to semi-inclusive hadron production in electron-positron annihilation. The calculation of the single-particle inclusive crosssection in time-like kinematics is performed in Mellin $N$-space and uses an algorithmic evaluation of inclusive phase-space integrals, based on the unitarity cutting rules and integration-by-parts. We obtain splitting functions and coefficient functions up to second order in the strong coupling $\alpha_{s}$. Our results are in agreement with earlier calculations in the literature by Rijken and van Neerven.
\end{abstract}




\section{Introduction}

The direct observation of hadron production in $e^{+} e^{-}$-annihilation offers unique ways to test predictions of QCD and has been measured in the past especially by the LEP experiments, see Ref. [1,2]. These data allow in particular for studies of single hadron production, fragmentation functions and their scaling violations. They also offer the possibility for determinations of the strong coupling constant $\alpha_{\mathrm{s}}$ at the scale $M_{Z}$.

In parallel to the deep-inelastic scattering (DIS) of leptons on hadrons, the fragmentation of the partons produced in the hard scattering process $e^{+} e^{-} \rightarrow q, \bar{q}, g$ into hadrons depends on the hadron's scaled momentum $x$. Perturbative QCD predicts the scale dependence and evolution of the scattering process, making higher order corrections mandatory for precision analyses. In the past, the next-to-leading order (NLO) results have been obtained in Refs. [3-6], while the coefficient functions at two loops, necessary for a next-to-next-to-leading order (NNLO) analysis were calculated [7-9]. In order to complete this program, the three-loop QCD predictions for the splitting function in time-like kinematics are required. The splitting functions governing the NNLO evolution of flavor non-singlet fragmentation distributions at third-order have recently been reported [10], while the corresponding singlet quantities are still missing.

Our motivation for the present paper is at least two-fold. First of all, given that $e^{+} e^{-}$-annihilation is of particular interest for the physics analyses of LEP data or a future ILC, we would like to provide an independent cross-check on the computation of Rijken and van Neerven [7-9]. Secondly, we perform a first application of a new innovative method [11] to calculate higher order QCD corrections to single-particle inclusive observables directly in Mellin $N$-space. It is worth emphasizing that the present method extends well beyond the framework of the operator product expansion (OPE) used in DIS [12-16]. This latter point is, in a more general perspective, rather important as it will allow for the efficient calculation of QCD corrections to many single scale observables. This can be done for Mellin moments either at fixed $N$ analogous to DIS calculations of Refs. [17-20] or by means of well established summation techniques [21] when full control over the analytic $N$ dependence is kept.

Finally, the present paper provides the means to thoroughly study the relation between the time-like QCD corrections to inclusive hadron production in $e^{+} e^{-}$annihilation and their spacelike counterparts, the DIS structure functions. At the leading order (LO) the Gribov-Lipatov relation [22] suggests simple relations between the splitting functions in both kinematics, which do not hold beyond LO [3,23], see also Refs. [24-26]. Yet, the space- and time-like cases are related by an analytic continuation in $x$ which has recently been used to obtain the NNLO flavor non-singlet time-like splitting functions [10]. In the present paper, we have also calculated in $d=4-2 \varepsilon$ di-

mensions so-far unknown terms at higher orders in $\varepsilon$ for the corresponding coefficient functions in $e^{+} e^{-}$-annihilation. These terms were subsequently used to check the analytical continuation between processes with space- and time-like kinematics [10].

The outline of the article is as follows. In Section 2 we set up the notation and the formalism for calculating QCD corrections to fragmentation functions. We also discuss the definition of the 
(time-like) splitting functions and respective coefficient functions through order $\alpha_{\mathrm{s}}^{2}$. Section 3 briefly explains the method to calculate Feynman diagrams in Mellin $N$-space for processes with time-like kinematics, with all details on the necessary master integrals and reductions given in Appendix A. In Section 4 we present our results for the fragmentation functions $F_{T}, F_{L}$ and $F_{A}$ through order $\alpha_{\mathrm{s}}^{2}$ and with details on the mass factorization to extract the splitting and coefficient functions. The lengthy full expressions are deferred to Appendix B ( $N$-space) and Appendix C ( $x$-space). We summarize in Section 5 .

\section{The setup}

The subject of our calculation is the single-particle inclusive $e^{+} e^{-}$-annihilation, i.e. the process

$$
e^{+}\left(k_{1}\right)+e^{-}\left(k_{2}\right) \rightarrow V(q) \rightarrow H\left(p_{H}\right)+X
$$

where $V$ is a vector boson, i.e. $V=\gamma, Z$, and $\left(k_{1}+k_{2}\right)^{2}=q^{2}=Q^{2}>0$ is its (time-like) fourmomentum squared. The observed hadron with momentum $p_{H}$ is denoted by $H$ and $X$ stands for any hadronic final states allowed by quantum number conservation.

Our goal is the derivation of the normalized double differential cross-section for the reaction in Eq. (1),

$$
\frac{1}{\sigma_{\text {tot }}} \frac{d^{2} \sigma^{H}}{d x d \cos \theta}=\frac{3}{8}\left(1+\cos ^{2} \theta\right) F_{T}(x)+\frac{3}{4} \sin ^{2} \theta F_{L}(x)+\frac{3}{4} \cos \theta F_{A}(x),
$$

in perturbation theory, and including the quantum corrections to the fragmentation functions $F_{T}, F_{L}$ and $F_{A}$ through order $O\left(\alpha_{\mathrm{s}}^{2}\right)$ in the strong coupling. In Eq. (2) $\sigma^{H}$ denotes the cross-section for producing the hadron $H, x$ is its scaled momentum fraction:

$$
x=\frac{2 p_{H} \cdot q}{Q^{2}}, \quad 0 \leq x \leq 1,
$$

and $\theta$ denotes the polar angle between the hadron and electron beam directions. The (total) fragmentation functions $F_{T}, F_{L}$ and $F_{A}$ in Eq. (2) originate from the transverse or longitudinal polarization states of the intermediate vector boson $(\gamma, Z)$ or from parity violation of the electroweak interaction. Upon integration over $\theta$ and $x$ the asymmetric contribution $F_{A}$ cancels and one arrives at the total cross-section integral

$$
\frac{1}{2} \int_{0}^{1} d x x \frac{1}{\sigma_{\mathrm{tot}}} \frac{d \sigma^{H}}{d x}=\frac{\sigma_{T}}{\sigma_{\mathrm{tot}}}+\frac{\sigma_{L}}{\sigma_{\mathrm{tot}}}=1
$$

which represents the energy-momentum sum-rule.

The evaluation of the fragmentation functions $F_{T}, F_{L}$ and $F_{A}$ in perturbative QCD is based on factorization. Up to power corrections suppressed by the hard scale $Q$, one can write the desired hadron level observables $F$ as a convolution of collinearly renormalized, parton level cross- 
sections $\hat{\mathcal{F}}$ with non-perturbative fragmentation distributions $D$. The explicit form of these relations is:

$$
\begin{aligned}
& F_{I}(x)=\sigma_{\text {tot }}^{(0)}\left(Q^{2}\right)\left[\hat{\mathcal{F}}_{I, q} \otimes D_{q \rightarrow H}^{\mathrm{s}}+\hat{\mathcal{F}}_{I, g} \otimes D_{g \rightarrow H}\right]+\sum_{h=1}^{n_{f}} \sigma_{h}^{(0)}\left(Q^{2}\right) \hat{\mathcal{F}}_{I, \mathrm{~ns}} \otimes D_{h \rightarrow H}^{\mathrm{ns},+}, \\
& F_{A}(x)=\sum_{h=1}^{n_{f}} A_{h}^{(0)}\left(Q^{2}\right) \hat{\mathcal{F}}_{A, \mathrm{~ns}} \otimes D_{h \rightarrow H}^{\mathrm{ns},-},
\end{aligned}
$$

where $I=T, L$ and the symbol $\otimes$ stands for the convolution integral,

$$
[f \otimes g](z)=\int_{0}^{1} d x \int_{0}^{1} d y f(x) g(y) \delta(z-x y) .
$$

The factors $\sigma_{\text {tot }}^{(0)}$ and $A_{h}^{(0)}$ are respectively the LO total cross-section and asymmetry factor for $e^{+} e^{-} \rightarrow$ hadrons. Moreover,

$$
\sigma_{\text {tot }}^{(0)}\left(Q^{2}\right)=\sum_{h=1}^{n_{f}} \sigma_{h}^{(0)}\left(Q^{2}\right) .
$$

The explicit expressions for $\sigma_{h}^{(0)}$ and $A_{h}^{(0)}$ can be found in Ref. [9].

The distributions $D$ for a non-singlet quark (of flavor $h$ ), a singlet quark $(q)$ or gluon $g$ fragmenting to the observed hadron $H$ are non-perturbative objects that are extracted from experimental data at low scales $Q$ of order $1 \mathrm{GeV}$ and evolved to high scales by means of the time-like Dokshitzer-Gribov-Lipatov-Altarelli-Parisi evolution equations [22, 27-29].

As discussed above, the time-like splitting functions are presently known to NLO accuracy in the flavor singlet and to NNLO in the non-singlet case [10]. Thus far, this has permitted the determination of the fragmentation distributions with next-to-leading logarithmic (NLL) accuracy. Examples of sets of LL and NLL accuracy are [30-33]. In case the hadron $H$ contains a heavy flavor, e.g. $b$-quark, the fragmentation distributions develop an additional perturbative component, a so-called 'perturbative fragmentation function' for the heavy quark [34]. That function allows the resummation of large logarithms of the ratio of the quark mass and the hard scale; it is currently known to NNLO $[35,36]$ which allows for the extraction of heavy quark fragmentation distributions with NNLL accuracy provided all three-loop time-like splitting functions are available.

The relation between the fragmentation distributions introduced above and the respective distributions for specific flavors are,

$$
\begin{aligned}
D_{h \rightarrow H}^{\mathrm{ns},+} & =D_{h \rightarrow H}+D_{\bar{h} \rightarrow H}-D_{q \rightarrow H}^{(S)}, \\
D_{h \rightarrow H}^{\mathrm{ns},-} & =D_{h \rightarrow H}-D_{\bar{h} \rightarrow H}, \\
D_{q \rightarrow H}^{\mathrm{s}} & =\frac{1}{n_{f}} \sum_{h=1}^{n_{f}}\left[D_{h \rightarrow H}+D_{\bar{h} \rightarrow H}\right],
\end{aligned}
$$

where $q$ denotes any generic 'quark' flavor, while $h$ stands for a specific quark flavor. The nonsinglet distributions evolve with the corresponding combinations of splitting functions [10] (see also Eqs. (30)-(34) in Ref. [13]). 
Next we turn our attention to the evaluation of the hard partonic cross-sections $\hat{\mathcal{F}}$ appearing in Eq. (5); the explicit results for these functions are discussed in Appendices B and C. The coefficient functions $\hat{\mathcal{F}}$ are related to the partonic equivalents $\mathcal{F}$ of the corresponding observables $F$. For the construction of the 'bare' functions $\mathcal{F}$ one replaces the observed hadron $H$ with an on-shell massless parton that can be $q, \bar{q}$ or $g$. Clearly, due to the incomplete inclusiveness of the observable (1), the partonic cross-sections $\mathcal{F}$ will contain additional collinear singularities, i.e. these functions are divergent even after the usual UV renormalization. To that end one performs a so-called 'collinear renormalization' or 'mass factorization'. One factorizes the collinear singularities and effectively absorbs them into the fragmentation distributions $D$. As an intermediate regularization we work in $d=4-2 \varepsilon$ dimensions [37-40]. In $z$-space and in the $\overline{\mathrm{MS}}$ scheme [41], these relation take the form (with $I=T, L$ and $J=T, L, A$ ):

$$
\begin{aligned}
\mathcal{F}_{J, \mathrm{~ns}}(\varepsilon, z) & =\hat{\mathcal{F}}_{J, \mathrm{~ns}}(z) \otimes \Gamma_{q q}^{\mathrm{ns}}(\varepsilon, z), \\
\mathcal{F}_{I, \mathrm{~s}}(\varepsilon, z) & =\hat{\mathcal{F}}_{I, \mathrm{~s}}(z) \otimes \Gamma_{q q}^{\mathrm{s}}(\varepsilon, z)+n_{f} \hat{\mathcal{F}}_{I, g}(z) \otimes \Gamma_{q g}(\varepsilon, z), \\
\mathcal{F}_{I, g}(\varepsilon, z) & =2 \hat{\mathcal{F}}_{I, q}(z) \otimes \Gamma_{g q}(\varepsilon, z)+\hat{\mathcal{F}}_{I, g}(z) \otimes \Gamma_{g g}(\varepsilon, z),
\end{aligned}
$$

and in Section 4 we explicitly present the corresponding expression in $N$-space. The form of the collinear counter-terms $\Gamma$ is universal, i.e. they contain only the time-like splitting functions. In the $\overline{\mathrm{MS}}$ scheme, and in terms of the bare strong coupling, their explicit expressions can be found in Eqs. (4.25)-(4.30) of Ref. [9] and we do not repeat them here ${ }^{1}$.

The partonic scaling variable $z$ appearing in Eq. (9) is the normalized energy fraction of the observed parton defined with respect to the total four-momentum $q$ of the $e^{+} e^{-}$system:

$$
z=\frac{2 p \cdot q}{Q^{2}}, \quad 0 \leq z \leq 1
$$

This definition of $z$ is consistent with the requirement that all partons are massless (see below).

$\hat{\mathcal{F}}$ in Eq. (9) above are the (finite) dimensionless partonic cross-sections with the collinear (or mass) singularities factorized in the $\overline{\mathrm{MS}}$ scheme. We have suppressed the explicit dependence on the factorization scale $\mu_{F}$; throughout this paper we set it equal to the hard scale $\mu_{F}^{2}=Q^{2}$. Moreover, all functions appearing in Eq. (9) are expressed in terms of the renormalized strong coupling. The relation between the bare $\alpha_{\mathrm{s}}$ and the renormalized $\alpha_{\mathrm{s}}\left(\mu_{R}\right)$ couplings reads:

$$
\frac{\alpha_{\mathrm{s}}}{4 \pi} S_{\varepsilon}=\frac{\alpha_{\mathrm{s}}\left(\mu_{R}\right)}{4 \pi}\left(1-\frac{\alpha_{\mathrm{s}}\left(\mu_{R}\right)}{4 \pi} \frac{\beta_{0}}{\varepsilon}+O\left(\alpha_{\mathrm{s}}^{2}\left(\mu_{R}\right)\right)\right) .
$$

The factor $S_{\varepsilon}=\exp \left(\varepsilon\left\{\ln (4 \pi)-\gamma_{\mathrm{e}}\right\}\right)$, where $\gamma_{\mathrm{e}}$ denotes the Euler-Mascheroni constant, is an artifact of dimensional regularization [37-40] kept out of the coefficient functions and anomalous dimensions in the $\overline{\mathrm{MS}}$ scheme. Also, we have

$$
\beta_{0}=\frac{11}{3} C_{A}-\frac{4}{3} T_{R} n_{f}
$$

\footnotetext{
${ }^{1}$ We would only like to caution the reader about the different notations for the definition of $\varepsilon$ and the normalization of the splitting functions used in these papers. In addition, the conventions for these counter-terms are the transposed of what is used in the more current literature.
} 
which is the $O\left(\alpha_{\mathrm{s}}^{2}\right)$ coefficient of the QCD $\beta$-function, $C_{A}=3, T_{R}=1 / 2$ are the QCD color factors, $n_{f}$ denotes the number of light fermion flavors and $\mu_{R}$ stands for the renormalization scale. To simplify our expressions we will work with $\mu_{R}^{2}=Q^{2}$. If desired, the explicit dependence on the arbitrary renormalization scale can be easily restored.

To completely specify our observables, we have to clarify how the bare partonic cross-sections are defined (and calculated). This is done next in Section 3 .

\section{The method}

\subsection{General considerations}

Similarly to the DIS case, the bare partonic differential distribution $\mathcal{F}$ for the process Eq. (1) can be written as a product of a leptonic and hadronic tensors. The hadronic tensor $W_{\mu \nu}$ is proportional to the amplitude squared of the decaying vector boson, and depends in particular on the coupling of the latter to the fermion current. Throughout this paper we will only consider the case of massless quarks $^{2}$. In this case, as was detailed in Ref. [9], the calculation of the inclusive QCD corrections to the corresponding coefficient functions is independent of the vector boson being vector (V) or axial-vector (A) type. For its evaluation it is therefore sufficient to consider the decay of a vector boson $V$ that couples to the fermion current as $\gamma^{\mu}\left(1-\gamma^{5}\right)$.

The bare hadronic tensor $W_{\mu \nu}$ depends on two momenta: the one of the decaying vector boson $q$ and the momentum of the observed parton $p$. Following the usual arguments for Lorentz and $\mathrm{CP}$ invariance one can show that $W_{\mu \nu}$ can be parameterized precisely with the three fragmentation functions $\mathcal{F}_{T, L, A}$ introduced in Eq. (9). For the V-A couplings mentioned above (see [9] for the general case), these three functions can be expressed as projections of the hadronic tensor $W_{\mu v}$ (with $d=4-2 \varepsilon$ ):

$$
\begin{aligned}
\mathcal{F}_{T}(z, \varepsilon) & =\frac{1}{d-2}\left(-2 \frac{p \cdot q}{q^{2}} W_{\mu}^{\mu}-\frac{2}{p \cdot q} p^{\mu} p^{v} W_{\mu v}\right) \\
\mathcal{F}_{L}(z, \varepsilon) & =\frac{1}{p \cdot q} p^{\mu} p^{v} W_{\mu \nu} \\
\mathcal{F}_{A}(z, \varepsilon) & =-\frac{1}{q^{2}} \frac{2}{(d-2)(d-3)} i \varepsilon^{\mu v \alpha \beta} p_{\alpha} q_{\beta} W_{\mu \nu} .
\end{aligned}
$$

Our goal in this paper is to calculate the fragmentation functions $\mathcal{F}_{T, L, A}$ including the coefficient functions of order $\alpha_{s}^{2}$. In fact, we even obtain the terms up to $\alpha_{s}^{2} \varepsilon^{2}$ in the mass-factorization, some of which we have used to check the analytical continuation of the space-like DIS results to the time-like region [10]. Terms of order $\alpha_{\mathrm{s}}^{2} \varepsilon^{2}$ would also be needed in a future evaluation of the order $\alpha_{\mathrm{s}}^{4}$ corrections to $\mathcal{F}_{T, L, A}$ (see also Eqs. (26)-(39) for more details on that point).

\footnotetext{
${ }^{2}$ Mass effects are known analytically through NLO [6] and, based on a numerical study, through NNLO [42, 43].
} 
Next, we explain how to construct the contributions of order $\alpha_{\mathrm{s}}^{k}$ to the hadronic tensor $W_{\mu \nu}$. This tensor contains the contributions from all diagrams for the processes,

$$
V(q) \rightarrow h(p)+h_{1}\left(p_{1}\right)+\ldots+h_{c}\left(p_{c}\right), \quad c=1 \ldots k+1
$$

of the decay of the vector boson $V$ with momentum $q$ to a set of particles $h, h_{1}, \ldots, h_{c}$. The different final states (labeled by the number $c$ of unobserved particles in that state) represent the contributions from the various physical cuts to the single-particle inclusive observable. After the Feynman diagrams contributing to each particular physical cut have been constructed and appropriately added, one has to perform the required virtual and/or (real) phase space integrations.

The approach we pursue for the evaluation of the required Feynman integrals differs significantly from the calculation in [7-9]. Our approach is based on the application of algebraic relations based on integration by parts (IBP) [37,44-46] to cross-sections and it is performed directly in Mellin $N$-space [11].

Our starting point is Eq. (13); in the following we will use $\mathcal{F}$ to denote any one of the three bare cross-sections $\mathcal{F}_{T, L, A}$. Each one of the functions $\mathcal{F}$ has a perturbative expansion in terms of the bare $^{3}$ strong coupling,

$$
\mathcal{F}(n, \varepsilon)=\sum_{k=0}^{\infty}\left(\frac{\alpha_{s}}{4 \pi} S_{\varepsilon}\right)^{k} \mathcal{F}^{(k)}(n, \varepsilon) .
$$

Throughout this paper, the letter $n$ will be reserved for the Mellin variable of any function of $z$ defined through

$$
f(n)=\int_{0}^{1} d z z^{n} f(z) .
$$

with $n \geq 0$. In particular, the total integral of a function corresponds to $n=0$. This definition of the Mellin variable is the most natural choice for the calculational procedures detailed in the following. We will, however, present the final results for the corresponding finite partonic cross-sections $\hat{\mathcal{F}}$ in terms of the conventional Mellin variable $N$ defined through

$$
\mathcal{F}(N, \varepsilon)=\int_{0}^{1} d z z^{N-1} \mathcal{F}(z, \varepsilon) .
$$

In view of the additional factor of $z$ in the partonic equivalent of Eq. (4) the relation between the two variables is

$$
n=N-2 \text {. }
$$

Each function $\mathcal{F}^{(k)}(n, \varepsilon)$ with $k \geq 0$ contains contributions from a number of terms, corresponding to the different physical cuts of the process $V \rightarrow h+X$ at order $\alpha_{\mathrm{s}}^{k}$ :

$$
\mathcal{F}^{(k)}(n, \varepsilon)=\sum_{c=1}^{k+1} \mathcal{F}_{(c)}^{(k)}(n, \varepsilon) .
$$

\footnotetext{
${ }^{3}$ We find it more convenient to present results in terms of the bare coupling instead of the renormalized one, the reason being that we work with massless on-shell partons. Thus we only need UV renormalization, i.e. the one related to the coupling Eq. 111.
} 
The functions $\mathcal{F}_{(c)}^{(k)}(n, \varepsilon)$ contain the full contributions from the process $V \rightarrow h+X$ at order $\alpha_{\mathrm{s}}^{k}$ where the inclusive final state $X$ contains $c$ unresolved partons. As described in Ref. [11], the construction of the functions $\mathcal{F}_{(c)}^{(k)}(n, \varepsilon)$ consists of the following steps:

1. One constructs all contributing Feynman diagrams. The integrations over the virtual momenta $\prod_{i} \frac{d^{d} k_{i}}{(2 \pi)^{d}}$ are assumed implicit in the diagrams.

2. One constructs the corresponding contribution to the tensor $W_{\mu \nu}$ by adding all relevant amplitudes squared, and with the appropriate symmetry factors included.

3. The above result is contracted with the appropriate tensor constructed from the $d$-dimensional metric and the momenta $p$ and $q$ as follows from Eq. (13).

4. The Lorentz scalar constructed this way is integrated over the full phase-space of the $c+1$ partons (i.e. one also integrates over the full phase-space of the 'observed' parton). The measure for this integration is:

$$
d \Phi=(2 p \cdot q)^{n}(2 \pi)^{d} \delta\left(q-p-p_{1}-\ldots-p_{c}\right) \prod_{i=0}^{c} \frac{d^{d} p_{i}}{(2 \pi)^{d-1}} \delta\left(p_{i}^{2}\right) .
$$

We have defined $p_{0} \equiv p$, and we have set $Q^{2}=1$ for simplicity. The exact dependence on $Q^{2}$ can be easily restored on dimensional grounds. The origin of the 'Mellin propagator' $2 p \cdot q$ is explained at the end of this Subsection.

5. One applies the IBP identities to reduce each term (generally containing integrations over both real and virtual momenta) to a linear combination of a small number of independent 'master' integrals. As a rule, at order $\alpha_{\mathrm{s}}^{k}$ and for each particular cut $c$, one needs to construct and solve more than one IBP reduction; the number of the required reductions corresponds to the number of independent topologies for each set $(k, c)$. The $\delta$-functions from the realphase space are dealt with along the lines of Ref. [47], while the Mellin propagator is treated along the lines of Ref. [11].

6. Each master integral is a function of the Mellin variable $n$. Its $n$-dependence can be completely extracted with the help of the difference equation the masters satisfy. The difference equations are obtained from the solutions to the IBP reduction (see also $[16,48]$ for related discussions in the DIS case).

7. One has to supply appropriate initial conditions for specifying the solutions of the difference equations. The most suitable choice is to evaluate the value of the masters at $n=0$. This choice corresponds to the total integral of each master over $z$. Therefore the initial conditions are pure, $\varepsilon$-dependent numbers.

8. Following [11], we 'partial fraction' by performing an additional summation over $n$ of the terms containing a propagator of the type $\sim 1 /(1-2 p \cdot q)$. This propagator is not linearly 
independent from the 'Mellin propagator' $2 p \cdot q$ as it merely shifts the effective $n$ in complete analogy to the DIS case, see e.g. Refs. $[13,48]$.

For the evaluation of the transverse and the longitudinal functions it is sufficient to take the matrix $\gamma^{5}$ as anti-commuting in $d$-dimensions. Special care is, however, needed for the evaluation of the asymmetric contribution $\mathcal{F}_{A, \text { ns }}$.

For the evaluation of $\mathcal{F}_{A, \text { ns }}$ we follow the prescription of Larin [49]. The details about the implementation can be found e.g. in $[13,14]$. In short, there are two important features: First, one replaces the axial-vector coupling $\gamma^{\mu} \gamma^{5}$ with the $d$-dimensional completely antisymmetric tensor $\varepsilon^{\mu \rho \sigma \tau} \gamma_{\rho} \gamma_{\sigma} \gamma_{\tau}$ and then uses its contraction properties with the second $\varepsilon$-tensor appearing in Eq. (13) to reduce it to combinations of the $d$-dimensional metric tensor. Second, one has to multiply the resulting expression with additional renormalization constants which restore the axial Ward identity in the $\overline{\mathrm{MS}}$-scheme. These constants have an expansion in the renormalized coupling $\alpha_{\mathrm{s}}$ and in powers of $\varepsilon$. They have been computed to three-loops in [50] and take the following form:

$$
\begin{aligned}
Z_{5}= & 1+\frac{\alpha_{s}\left(\mu_{R}\right)}{4 \pi} C_{F}\left\{-4-10 \varepsilon+\left(-22+2 \zeta_{2}\right) \varepsilon^{2}+o\left(\varepsilon^{3}\right)\right\} \\
& +\left(\frac{\alpha_{s}\left(\mu_{R}\right)}{4 \pi}\right)^{2}\left\{22 C_{F}^{2}-\frac{107}{9} C_{A} C_{F}+\frac{2}{9} n_{f} C_{F}\right. \\
& \left.+\left(\left(132-48 \zeta_{3}\right) C_{F}^{2}+\left(-\frac{7229}{54}+48 \zeta_{3}\right) C_{A} C_{F}+\frac{331}{27} n_{f} C_{F}\right) \varepsilon+o\left(\varepsilon^{2}\right)\right\}+o\left(\alpha_{\mathrm{s}}^{3}\right) \\
Z_{A}= & 1+\left(\frac{\alpha_{s}\left(\mu_{R}\right)}{4 \pi}\right)^{2} \frac{1}{\varepsilon}\left\{\frac{22}{3} C_{A} C_{F}-\frac{4}{3} n_{f} C_{F}\right\}+o\left(\alpha_{\mathrm{s}}^{3}\right) .
\end{aligned}
$$

We would like to conclude this Subsection with a comment on the origin of the 'Mellin propagator' $P_{M}=2 p \cdot q$ appearing in Eq. (20). As was detailed in [11], to construct the bare distribution $\mathcal{F}(z, \varepsilon)$ in $z$-space, one has to integrate over the full phase space of all final states particle and insert the additional factor $\delta(z-2 p \cdot q)$. If one Mellin-transforms this expression before the required phase-space and virtual integrations are performed, one gets schematically (see also Eqs. (10), (20):

$$
\begin{aligned}
\mathcal{F}(n, e) & =(\ldots) \times \int_{0}^{1} d z z^{n} \delta(z-2 p \cdot q) \\
& =(\ldots) \times(2 p \cdot q)^{n}
\end{aligned}
$$

i.e. the factor of the 'Mellin propagator' raised to a symbolic power $n$ that appears in Eq. (20). The factor $(.$.$) stands for the various propagators (including possibly additional powers of P_{M}$ ), the measures for the real and/or virtual integrations, etc., but contains no dependence on $z$ or $n$. This procedure is completely analogous but more general than the corresponding DIS case [12-16], which relies on the OPE and the method of projection to directly expand propagators in powers of $\left(2 p \cdot q / q^{2}\right)^{n}$. There Eq. (22) is effectively realized by mapping any Feynman diagram to Mellin moments with the help of a suitable projection operator [51,52].

In the following we will present the specifics of the implementation of the above procedure for the evaluation of the contributions at orders $\alpha_{\mathrm{s}}$ and $\alpha_{\mathrm{s}}^{2}$. 


\subsection{Order $O\left(\alpha_{\mathrm{s}}\right)$}

Here we discuss both the derivation and the results for all independent contributions at order $O\left(\alpha_{\mathrm{s}}\right)$. This order is the NLO result for the transverse and the asymmetric contributions, but the LO result for the longitudinal function, since it vanishes at order $O\left(\alpha_{\mathrm{s}}^{0}\right)$.

At order $O\left(\alpha_{\mathrm{s}}\right)$ in the perturbative expansion, one has to consider only two cuts: one corresponding to real gluon emission (where the final state is $(q \bar{q} g)$ ) and the one with the virtual correction to the $V q \bar{q}$ vertex (with the final state being $(q \bar{q})$ ). The evaluation of these two contributions is fairly different. We discuss first the contribution from the virtual corrections.

From the kinematics of the tree-level decay process $V \rightarrow q \bar{q}$ it is clear that the contribution of the purely virtual corrections to the functions $\mathcal{F}$ are of the type const $(\varepsilon) \delta(1-z)$ in $z$-space which corresponds to a $n$-independent constant in $n$-space. Therefore, the contribution from this cut is completely determined by (twice) the real part of the time-like form-factor [53-55].

All non-trivial $n$-dependence at that order comes from the real-emission diagrams. The corresponding diagrams can be found in Ref. [9] and we do not repeat them here. As was outlined in the previous Subsection, for the evaluation of all contributions $(T, L, A)$ for both a quark and a gluon, one needs to construct a single topology consisting of five 'propagators' (see also [11]). One of the arguments, of course, corresponds to the Mellin propagator $2 p \cdot q$. We performed the required IBP reductions and obtained a single $n$-dependent master integral, which can be found e.g. in [11].

To perform the reductions resulting from the IBP identities, we have used the program AIR [56] which is an implementation of the so-called Laporta algorithm [57-60] in MAPLE. AIR contains also a routine which conveniently and automatically maps the constructed diagrams into the master integrals of the reductions (a single master at this order). Following this simple procedure, one can map the whole problem of the evaluation of any one of the functions $\mathcal{F}$ at order $\alpha_{\mathrm{s}}^{1}$ to the single master multiplied by a rational function of $n$ and $\varepsilon$. As discussed above there is another type of contributions containing a propagator that is not linearly independent of the Mellin propagator $P_{M}=2 p \cdot q$, i.e. contributions of the form:

$$
\text { (..) } \frac{P_{M}^{n}}{1-P_{M}}=\sum_{k=0}^{\infty}(\ldots) P_{M}^{n+k}
$$

where dots stand for any other, linearly independent propagator and the integration measure over the real momenta. Since the term on the right hand side of Eq. (23) is of the usual form, the results from the solutions to the IBP reduction can be applied (with $n$ replaced by $n+k$ ) and then summed

over $k$. To illustrate that point, we present the corresponding term of the order $\alpha_{\mathrm{s}}^{1}$ contribution to the function $\mathcal{F}_{q}^{T}$ :

$$
\begin{aligned}
& C_{F} \sum_{k=n}^{\infty} \varepsilon(1-\varepsilon)\left(4 \varepsilon^{3}-4 \varepsilon^{2} k-54 \varepsilon^{2}+31 \varepsilon k+74 \varepsilon+\varepsilon k^{2}-4 k^{2}-20 k-24\right) \\
& \times \frac{\Gamma(-\varepsilon)^{2} \Gamma(k+1-2 \varepsilon)}{\Gamma(2-2 \varepsilon) \Gamma(4-3 \varepsilon+k)},
\end{aligned}
$$


which can be easily summed up in terms of $\Gamma$-functions. This way, one arrives at a very compact result for the bare $(T, L, A)$ partonic cross-sections at order $\alpha_{\mathrm{s}}$ valid to all orders in $\varepsilon$. The explicit results, expanded to sufficient powers in $\varepsilon$, can be found in Section 4

\subsection{Order $O\left(\alpha_{\mathrm{s}}^{2}\right)$}

At this order one has to consider three different cuts: double-virtual corrections with final state $(q, \bar{q})$, one-loop virtual corrections to the final state $(q, \bar{q}, g)$ and, finally, the cut with double real emission. The latter consists of the following final states: $(q, \bar{q}, g, g) ;(q, \bar{q}, q, \bar{q})$ and $\left(q, \bar{q}, q^{\prime}, \bar{q}^{\prime}\right)$. Depending on the gauge choice for the polarization of the external gluons one may also have to consider external ghosts. Again the corresponding diagrams can be found in Ref. [9] and we are only interested in the evaluation of the diagrams with real emissions since the diagrams with two-loop virtual corrections produce only constant terms in Mellin $n$-space. These purely virtual contributions can be obtained from the one- and two-loop time-like form-factor [53-55].

To cover all possible diagrams for the evaluation of both quark and gluon production we construct seven topologies for the double real emission cut, and five topologies for the real-virtual cut. After symmetry considerations, we arrive at a total of six real-real and five different real-virtual masters. These $n$-dependent masters satisfy difference equations that can be read off the completed reductions. For completeness, we have presented both the definitions of the masters and the difference equations they satisfy in Appendix A.

As can be seen there, the structure of the equations is simple. The simplest masters decouple and satisfy homogeneous equations, while the more complicated ones satisfy first order difference equations with the non-homogeneous part comprised by simpler, explicitly known masters. Such first order equations can be easily solved in closed form to all orders in $n$ and $\varepsilon$, see e.g. Ref. [13]. If we pursue this approach, the most complicated solutions we encounter are Appel functions of unit arguments or hypergeometric functions of unit argument.

However, we decided to follow a different path. One can also obtain the solutions of the difference equations after an expansion in powers of $\varepsilon$ using the methods of symbolic summation and the packages Summer [61] and XSummer [62] in Form [63]. Then it is very easy to solve the equations this way given that previously even three-loop master integrals have been computed $[14,55,62]$. In this approach, one is required to supply the initial conditions (for $n=0$ ) beforehand. This is to be contrasted with the all-order in $\varepsilon$ calculation where the initial condition factorizes completely.

Only one master, $R_{2}(n)$ deserves special consideration. It formally satisfies a second order difference equation as can be seen from Eq. A.13). A closer inspection, however, reveals that it is a second order difference equation of defined parity (thus a $n-1$ term is absent). Therefore one can write this equation as a first order difference equation for a 'new variable' $n^{\prime}=n / 2$. In doing so we could solve the resulting difference equation in terms of ${ }_{7} F_{6}$-type functions of unit argument. These functions contain half-integer (and $n / 2$ ) parameters and are not simple to expand in $\varepsilon$. If one, however, solves this master as an expansion in powers of $\varepsilon$ no particular complications 
arise besides the fact that one has to supply two initial conditions (for $n=0$ and for $n=1$ ) for any second order equation.

Next we address the calculation of the initial conditions for the masters. Our first step is to perform new IBP reductions in each of the real-real and real-virtual topologies setting $n=0$ from the very beginning. From this fixed order reductions we obtain relations between the initial conditions for the masters integrals. In the end, we find that a total of seven are independent. Most of these integrals are very easy to compute directly to all orders in $\varepsilon$. The most involved is the initial condition $R_{6}(0)$ given in Eq. A.23). For its evaluation (up to weight 6 in values of the Riemann zeta-function) we have used the approach of Ref. [64] based on the optical theorem and the results of Ref. [65] for the higher order $\varepsilon$ terms of the non-planar three-loop two-point function. With the help of these additional fixed- $n$ runs we can derive a relation (see Eq. (A.23) between the values of the real master $R_{2}$ at $n=0$ and $n=1$ as mentioned above.

The last point that deserves special attention are terms $\sim 1 /(1-2 p \cdot q)$. 'Partial fractioning' of these leads to an additional sum as explained above, cf. Eq. (23). In performing the required symbolic summation we employ the following strategy. We explicitly separate sums from 1 to $\infty$ over $n$-independent terms, as we systematically ignore constant terms from the evaluation of the diagrams with real emissions as well as the constant terms from the purely virtual corrections. After we complete our evaluation we can restore these constant terms from the requirement that the total cross-section is reproduced (see Eq. (4)). We wish to emphasize, though, that this procedure is simply done to economize on the necessary algebra and by no means represents any principal drawback of our approach.

The above comment applies to the transverse and asymmetric partonic cross-sections. Since the longitudinal cross-sections do not contain any constant terms, this procedure uniquely fixes the missing constant contributions in the transverse functions. It also provides the coefficient of the $\delta$-function contributions to the asymmetric functions. To that end, one uses the fact that the difference of the transverse and asymmetric functions when expressed in $z$-space, does not contain $\delta$-functions and singular +-distributions. Equivalently, the difference of these functions vanishes in the 'soft' limit $N \rightarrow \infty$.

\section{Results}

Let us now present the results of the calculation. As explained above, mass factorization (or collinear renormalization) predicts a specific structure for the final result, which we write out up to second order in the strong coupling $a_{\mathrm{s}}=\alpha_{\mathrm{s}} /(4 \pi)$. Following the conventions from Eq. 15), we present the general structure of the result expanded in $\varepsilon$ directly in Mellin $N$-space. According to Eq. (17), the Mellin moments of the splitting functions are defined by

$$
P(N)=\int_{0}^{1} d z z^{N-1} P(z)=-\gamma(N),
$$


and we note the (conventional) sign for the relation of the splitting functions to the anomalous dimensions. In the following, all products are to be evaluated employing the algebra for harmonic sums $[13,61]$.

The zeroth-order contributions, with $\mathcal{F}_{T, \mathrm{q}}^{(0)}$ being suitably normalized, read

$$
\mathcal{F}_{T, \mathrm{q}}^{(0)}=c_{T, \mathrm{q}}^{(0)}=1, \quad \mathcal{F}_{T, \mathrm{~g}}^{(0)}=\mathcal{F}_{L, \mathrm{q}}^{(0)}=\mathcal{F}_{L, \mathrm{~g}}^{(0)}=0, \quad \mathcal{F}_{A, \mathrm{q}}^{(0)}=c_{A, \mathrm{q}}^{(0)}=1 .
$$

Note that not all functions are independent; on general grounds one can show that [9]:

$$
\begin{aligned}
\mathcal{F}_{T, \mathrm{q}} & =+\mathcal{F}_{T, \overline{\mathrm{q}}}, \\
\mathcal{F}_{A, \mathrm{q}} & =-\mathcal{F}_{A, \overline{\mathrm{q}}}, \\
\mathcal{F}_{A, \mathrm{~g}} & =0 .
\end{aligned}
$$

For the applications of the present study we present the amplitudes at the first order in $\alpha_{\mathrm{s}}$ up to terms of the order $\varepsilon^{2}$, yielding for $\mathcal{F}_{T}$

$$
\begin{aligned}
& \mathcal{F}_{T, \mathrm{q}}^{(1)}=-\frac{1}{\varepsilon} P_{\mathrm{qq}}^{(0)}+c_{T, \mathrm{q}}^{(1)}+\varepsilon a_{T, \mathrm{q}}^{(1)}+\varepsilon^{2} b_{T, \mathrm{q}}^{(1)}, \\
& \mathcal{F}_{T, \mathrm{~g}}^{(1)}=-\frac{2}{\varepsilon} P_{\mathrm{gq}}^{(0)}+c_{T, \mathrm{~g}}^{(1)}+\varepsilon a_{T, \mathrm{~g}}^{(1)}+\varepsilon^{2} b_{T, \mathrm{~g}}^{(1)},
\end{aligned}
$$

for the longitudinal $\mathcal{F}_{L}$

$$
\begin{aligned}
& \mathcal{F}_{L, \mathrm{q}}^{(1)}=c_{L, \mathrm{q}}^{(1)}+\varepsilon a_{L, \mathrm{q}}^{(1)}+\varepsilon^{2} b_{L, \mathrm{q}}^{(1)}, \\
& \mathcal{F}_{L, \mathrm{~g}}^{(1)}=c_{L, \mathrm{~g}}^{(1)}+\varepsilon a_{L, \mathrm{~g}}^{(1)}+\varepsilon^{2} b_{L, \mathrm{~g}}^{(1)}
\end{aligned}
$$

and for the asymmetric $\mathcal{F}_{A}$

$$
\mathcal{F}_{A, \mathrm{~ns}}^{(1)}=-\frac{1}{\varepsilon} P_{\mathrm{qq}}^{(0)}+c_{A, \mathrm{q}}^{(1)}+\varepsilon a_{A, \mathrm{q}}^{(1)}+\varepsilon^{2} b_{A, \mathrm{q}}^{(1)} .
$$

Correspondingly, the $\alpha_{\mathrm{s}}^{2}$ contributions where the non-singlet and singlet quark amplitudes differ for the first time, are required up to order $\varepsilon$. These quantities are given by

$$
\begin{aligned}
\mathcal{F}_{T, \mathrm{~ns}}^{(2)}= & \frac{1}{2 \varepsilon^{2}}\left\{P_{\mathrm{qq}}^{(0)}\left(P_{\mathrm{qq}}^{(0)}+\beta_{0}\right)\right\}-\frac{1}{2 \varepsilon}\left\{P_{\mathrm{ns}}^{(1)+}+2 c_{T, \mathrm{q}}^{(1)} P_{\mathrm{qq}}^{(0)}\right\} \\
& +c_{T, \mathrm{~ns}}^{(2)}-a_{T, \mathrm{q}}^{(1)} P_{\mathrm{qq}}^{(0)}+\varepsilon\left\{a_{T, \mathrm{~ns}}^{(2)}-b_{T, \mathrm{q}}^{(1)} P_{\mathrm{qq}}^{(0)}\right\} \\
\mathcal{F}_{T, \mathrm{ps}}^{(2)}= & \frac{1}{2 \varepsilon^{2}}\left\{P_{\mathrm{qg}}^{(0)} P_{\mathrm{gq}}^{(0)}\right\}-\frac{1}{2 \varepsilon}\left\{P_{\mathrm{qq}}^{(1) \mathrm{s}}+c_{T, \mathrm{~g}}^{(1)} P_{\mathrm{qg}}^{(0)}\right\} \\
& +c_{T, \mathrm{ps}}^{(2)}-\frac{1}{2} a_{T, \mathrm{~g}}^{(1)} P_{\mathrm{qg}}^{(0)}+\frac{1}{2} \varepsilon\left\{2 a_{T, \mathrm{ps}}^{(2)}-b_{T, \mathrm{~g}}^{(1)} P_{\mathrm{qg}}^{(0)}\right\} \\
\mathcal{F}_{T, \mathrm{~g}}^{(2)}= & \frac{1}{\varepsilon^{2}}\left\{P_{\mathrm{gq}}^{(0)}\left(P_{\mathrm{qq}}^{(0)}+P_{\mathrm{gg}}^{(0)}+\beta_{0}\right)\right\}+\frac{1}{\varepsilon}\left\{P_{\mathrm{gq}}^{(1)}+2 c_{T, \mathrm{q}}^{(1)} P_{\mathrm{gq}}^{(0)}+c_{T, \mathrm{~g}}^{(1)} P_{\mathrm{gg}}^{(0)}\right\} \\
& +c_{T, \mathrm{~g}}^{(2)}-2 a_{T, \mathrm{q}}^{(1)} P_{\mathrm{gq}}^{(0)}-a_{T, \mathrm{~g}}^{(1)} P_{\mathrm{gg}}^{(0)}+\varepsilon\left\{a_{T, \mathrm{~g}}^{(2)}-2 b_{T, \mathrm{q}}^{(1)} P_{\mathrm{gq}}^{(0)}-b_{T, \mathrm{~g}}^{(1)} P_{\mathrm{gg}}^{(0)}\right\},
\end{aligned}
$$




$$
\begin{aligned}
\mathcal{F}_{L, \mathrm{~ns}}^{(2)}= & -\frac{1}{\varepsilon}\left\{c_{L, \mathrm{q}}^{(1)} P_{\mathrm{qq}}^{(0)}\right\}+c_{L, \mathrm{~ns}}^{(2)}-a_{L, \mathrm{q}}^{(1)} P_{\mathrm{qq}}^{(0)}+\varepsilon\left\{a_{L, \mathrm{~ns}}^{(2)}-b_{L, \mathrm{q}}^{(1)} P_{\mathrm{qq}}^{(0)}\right\} \\
\mathcal{F}_{L, \mathrm{ps}}^{(2)}= & -\frac{1}{\varepsilon}\left\{c_{L, \mathrm{~g}}^{(1)} P_{\mathrm{qg}}^{(0)}\right\}+c_{L, \mathrm{ps}}^{(2)}-\frac{1}{2} a_{L, \mathrm{~g}}^{(1)} P_{\mathrm{qg}}^{(0)}+\frac{1}{2} \varepsilon\left\{2 a_{L, \mathrm{ps}}^{(2)}-b_{L, \mathrm{~g}}^{(1)} P_{\mathrm{qg}}^{(0)}\right\} \\
\mathcal{F}_{L, \mathrm{~g}}^{(2)}= & \frac{1}{\varepsilon}\left\{2 c_{L, \mathrm{q}}^{(1)} P_{\mathrm{gq}}^{(0)}+c_{L, \mathrm{~g}}^{(1)} P_{\mathrm{gg}}^{(0)}\right\}+c_{L, \mathrm{~g}}^{(2)}-2 a_{L, \mathrm{q}}^{(1)} P_{\mathrm{gq}}^{(0)}-a_{L, \mathrm{~g}}^{(1)} P_{\mathrm{gg}}^{(0)} \\
+ & \varepsilon\left\{a_{L, \mathrm{~g}}^{(2)}-2 b_{L, \mathrm{q}}^{(1)} P_{\mathrm{gq}}^{(0)}-b_{L, \mathrm{~g}}^{(1)} P_{\mathrm{gg}}^{(0)}\right\} . \\
\mathcal{F}_{A, \mathrm{~ns}}^{(2)}= & \frac{1}{2 \varepsilon^{2}}\left\{P_{\mathrm{qq}}^{(0)}\left(P_{\mathrm{qq}}^{(0)}+\beta_{0}\right)\right\}-\frac{1}{2 \varepsilon}\left\{P_{\mathrm{ns}}^{(1)-}+2 c_{A, \mathrm{q}}^{(1)} P_{\mathrm{qq}}^{(0)}\right\} \\
& +c_{A, \mathrm{~ns}}^{(2)}-a_{A, \mathrm{q}}^{(1)} P_{\mathrm{qq}}^{(0)}+\varepsilon\left\{a_{A, \mathrm{~ns}}^{(2)}-b_{A, \mathrm{q}}^{(1)} P_{\mathrm{qq}}^{(0)}\right\}
\end{aligned}
$$

The results for the splitting and coefficient functions at order $O\left(\alpha_{\mathrm{s}}\right)$ and $O\left(\alpha_{\mathrm{s}}^{2}\right)$ are given both in $N$-space (Appendix B) and $x$-space (Appendix C). The precise definition of the various splitting functions can be found in Eqs. (30)-(34) in [13], see also [66] for a more detailed discussion on that point.

Several comments are in order: First of all, as we have performed the calculation in Mellin space, all $x$-dependence is recovered from the $N$-space results by an inverse Mellin transformation, which expresses these functions in terms of harmonic polylogarithms [67]. The inverse Mellin transformation exploits an isomorphism between the set of harmonic sums for even or odd $N$ and the set of harmonic polylogarithms (see also Appendix B). The algebraic procedure [13,67] is based on the fact that harmonic sums occur as coefficients of the Taylor expansion of harmonic polylogarithms.

Our results for the finite terms in $\varepsilon$ agree with the ones in [7-9]. We have found several misprints in these references and would like to take the opportunity to point out these typos in the original manuscript of Ref. [9] (employing the notation of the original reference). In Eq. (A.6) of Ref. [9] for $\left.\bar{c}_{T, q}^{\mathrm{NS},(2), \text { nid }}\right|_{H}$ there should be a replacement of the term

$$
C_{F}^{2}(1+z)\left(\ldots-3 \ln ^{2} z \ldots\right) \quad \longrightarrow \quad C_{F}^{2}(1+z)\left(\ldots-3 \ln ^{3} z \ldots\right),
$$

in Eq. (A.8) of Ref. [9] for $\bar{c}_{T, q}^{\mathrm{NS},(2), \text { id }}$ of the term

$$
\left(C_{F}^{2}-\frac{1}{2} C_{A} C_{F}\right)\left(\frac{24}{5 z^{2}}+\ldots\right) \ln z \quad \longrightarrow \quad\left(C_{F}^{2}-\frac{1}{2} C_{A} C_{F}\right)\left(\frac{24}{5 z}+\ldots\right) \ln z
$$

in Eq. (A.10) of Ref. [9] for $\bar{c}_{T, q}^{\mathrm{PS},(2)}$ of the term

$$
C_{F} T_{f}\left[\ldots+\frac{11}{6} \ln ^{3} z+\ldots \quad \longrightarrow \quad C_{F} T_{f}\left[\ldots+\frac{11}{6} \ln ^{3} z\right)+\ldots\right.
$$


and in Eq. (A.15) of Ref. [9] for $\bar{c}_{L, q}^{\mathrm{NS},(2), \text { id }}$ of the term

$$
\left(C_{F}^{2}-\frac{1}{2} C_{A} C_{F}\right)\left(32 S_{1,2}(1-z)+\ldots\right) \quad \longrightarrow \quad\left(C_{F}^{2}-\frac{1}{2} C_{A} C_{F}\right)\left(32 S_{1,2}(-z)+\ldots\right)
$$

Finally, in Eq. (17) of Ref. [8] for $\mathbf{C}_{A, q}^{\mathrm{NS}, \text { nid,(2) }}-\mathbf{C}_{T, q}^{\mathrm{NS}, \text { nid,(2) }}$ one should replace the term

$$
C_{F}^{2}\left(-\frac{24}{5 z^{2}}+\ldots\right) \ln z \quad \longrightarrow \quad C_{F}^{2}\left(-\frac{24}{5 z}+\ldots\right) \ln z
$$

Beyond the coefficient functions at order $O\left(\alpha_{\mathrm{s}}^{2}\right)$ we have also obtained the terms of higher order in the $\varepsilon$ expansion, specifically $a^{(1)}, b^{(1)}$ and $a^{(2)}$. In the non-singlet case, we have found them to agree with the predictions based on the analytical continuation proposed in [10]. However, as these expressions $\left(a^{(1)}, b^{(1)}\right.$ and $\left.a^{(2)}\right)$ are particularly lengthy and have no direct physical application, we refrain from writing them out explicitly here.

For future use and for uniformity of the notations, we present in the Appendices B and C also the explicit expressions for the one- and two-loop time-like splitting functions. Our calculations agree with the known results [3,4] (see also [66]). This statement, though, is subject to one qualification. As we are considering in Eq. (1) only the decay of a vector boson $V$, we have no access to the two-loop (time-like) splitting functions $P_{\mathrm{qg}}^{(1)}$ and $P_{\mathrm{gg}}^{(1)}$ with the set of Feynman diagrams considered. To do so, we would actually be required to compute also the decay of a (fictitious) classical scalar $\phi$ that couples directly only to the gluon field via $\phi G_{\mu \nu}^{a} G_{a}^{\mu \nu}$. In time-like kinematics, this approach has been used for instance to derive $P_{\mathrm{gg}}^{(1)}$ in [68]; see also Refs. $[15,16,18]$ for the analogous considerations in the space-like case. An inclusion of the $\phi G_{\mu \nu}^{a} G_{a}^{\mu \nu}$ coupling is straight forward and would allow for the determination of $P_{\mathrm{qg}}^{(1)}$ and $P_{\mathrm{gg}}^{(1)}$ (or rather its Mellin transform) to the desired two-loop accuracy.

\section{Summary}

We have calculated the $O\left(\alpha_{\mathrm{s}}^{2}\right)$ corrections to the transverse, longitudinal and asymmetric fragmentation functions for both quarks and gluons in semi-inclusive $e^{+} e^{-}$-annihilation to hadrons. Our calculation confirms the results of Rijken and van Neerven [7-9] and we have taken the opportunity to correct several typographical errors in these papers (see also [10]). Our results constitute a strong check on [7-9], in particular since we have used a rather different technology and obtained them directly in Mellin $N$-space following the proposal in [11]. Thus, our calculation represents the first example of a single-particle inclusive observable beyond the well established DIS framework [12-16] that is computed analytically in Mellin $N$-space.

The coefficient functions presented in this paper contain the NNLO corrections to the transverse and asymmetric fragmentation functions $\mathcal{F}_{T}$ and $\mathcal{F}_{A}$, and the NLO corrections to the longitudinal ones, $\mathcal{F}_{L}$. After the complete singlet three-loop time-like splitting functions become available (the 
non-singlet case has recently been reported [10]), one will be able to study light and heavy quark fragmentation at NNLO [34-36]. This important class of observables will allow precise extraction of fragmentation distributions from LEP data and is yet another motivation for the realization of the envisioned high-precision Giga- $Z$ option of the future ILC. Due to the process independence of the fragmentation distributions, they can be further applied to other processes like hadro- and photo-production.

In addition, the present paper provides the means to thoroughly study the relations between the time-like QCD corrections to inclusive hadron production in $e^{+} e^{-}$annihilation and their spacelike counterparts, the DIS structure functions. Our calculational approach easily allows us to obtain higher powers in $\varepsilon$ of the bare partonic cross-sections at order $O\left(\alpha_{\mathrm{s}}^{2}\right)$, which had not been computed before. Thus, we could provide important cross checks on the procedure of Ref. [10] based on an analytic continuation in $x$ between observables with space- and time-like kinematics. Of course, the aforementioned higher terms in $\varepsilon$ will also be needed for a future evaluation of the QCD corrections to $e^{+} e^{-}$-annihilation at order $O\left(\alpha_{\mathrm{s}}^{3}\right)$. Finally, one particularly appealing feature of the $N$-space approach is the small number of master integrals that have to be evaluated. Moreover, with the boundary conditions in $N$-space being kinematics independent, the corresponding integrals may also be of relevance in other circumstances. As a matter of fact, some of them had been considered before in a different context [64]. In the present paper we have extended these results to higher powers in $\varepsilon$.

Among the prospects for future developments and applications of our results and methods are explicit three-loop checks of the splitting and coefficient functions in semi-inclusive $e^{+} e^{-}$ annihilation, for instance by computations of fixed- $N$ Mellin moments. Also QCD corrections to many other single scale observables can be considered.

FORM files of our results can be obtained from the preprint server http://arXiv.org by downloading the source. Furthermore they are available from the authors upon request.

Acknowledgments: We are grateful to A. Vogt for useful discussions. A.M. acknowledges support by the Alexander von Humboldt Foundation. The work of S.M. has been supported in part by the Helmholtz Gemeinschaft under contract VH-NG-105.

Note added: The (un-)polarized coefficient functions up to two loops have recently been transformed to Mellin $N$-space in [70].

\section{Appendix A: Master Integrals}

In this Appendix, we present the complete list of master integrals, the corresponding difference equations in Mellin $n$-space and the respective boundary conditions. We omit the discussion of the so-called purely virtual contributions, which are known since long. For the calculations of two-loop form factors in QCD we refer to Refs. [54,55,69].

Let us start with the master integrals $\mathrm{V}_{1}(n), \ldots, \mathrm{V}_{5}(n)$ for the so-called real-virtual contribu- 
tions. These masters can be defined through the following object:

$$
\begin{aligned}
& {[i, \ldots, j]=} \\
& \quad \frac{e^{\left(3 \gamma_{\mathrm{e}} \varepsilon\right)}}{\pi^{4}} \int d^{d} k d^{d} p_{1} d^{d} p_{2} \delta\left(p_{1}^{2}\right) \delta\left(p_{2}^{2}\right) \delta\left(\left(q-p_{1}-p_{2}\right)^{2}\right)\left(2 q \cdot\left(q-p_{1}-p_{2}\right)\right)^{n} \frac{1}{P_{i} \ldots P_{j}},
\end{aligned}
$$

where $q \cdot q=1$ and the propagators are $P_{1}=k^{2}, P_{2}=\left(q-p_{1}+k\right)^{2}, P_{3}=(q-k)^{2}, P_{4}=\left(p_{1}+\right.$ $\left.p_{2}+k\right)^{2}, P_{5}=\left(p_{1}-k\right)^{2}, P_{6}=\left(p_{2}+k\right)^{2}, P_{7}=\left(q-p_{2}\right)^{2}$.

The $n$-dependent real-virtual masters are defined as: $\mathrm{V}_{1}(n)=[1,2], \mathrm{V}_{2}(n)=[1,3], \mathrm{V}_{3}(n)=$ $[1,4], \mathrm{V}_{4}(n)=[1,2,5], \mathrm{V}_{5}(n)=[1,2,5,6,7]$. These masters satisfy the following difference equations:

$$
\begin{aligned}
& (n+2-4 \varepsilon) \mathrm{V}_{1}(n)-(n+1-3 \varepsilon) \mathrm{V}_{1}(n-1)=0, \\
& (n+2-3 \varepsilon) \mathrm{V}_{2}(n)-(n+1-2 \varepsilon) \mathrm{V}_{2}(n-1)=0, \\
& (n+2-4 \varepsilon) \mathrm{V}_{3}(n)-(n+1-2 \varepsilon) \mathrm{V}_{3}(n-1)=0, \\
& (n+1-2 \varepsilon) \mathrm{V}_{4}(n)-n \mathrm{~V}_{4}(n-1)= \\
& -(1-3 \varepsilon)(1-2 \varepsilon) \frac{1}{\varepsilon} \mathrm{V}_{1}(n-1)+(1-2 \varepsilon)^{2} \frac{1}{\varepsilon} \mathrm{V}_{2}(n-1), \\
& (n-1-4 \varepsilon) \mathrm{V}_{5}(n)-(n-1-2 \varepsilon) \mathrm{V}_{5}(n-1)= \\
& -\frac{(1-3 \varepsilon)(1-2 \varepsilon)(n+1-4 \varepsilon)}{(n-3 \varepsilon)(n-1-3 \varepsilon)}\left(27 \varepsilon^{2}-11 n \varepsilon+5 \varepsilon-n+n^{2}\right) \frac{1}{\varepsilon^{2}} \mathrm{~V}_{1}(n-1) \\
& +\frac{(1-2 \varepsilon)^{2}(n-4 \varepsilon)(n+1-3 \varepsilon)}{n-2 \varepsilon} \frac{1}{\varepsilon^{2}} \mathrm{~V}_{2}(n-1)+(n-2 \varepsilon) \mathrm{V}_{4}(n-1)
\end{aligned}
$$

The boundary conditions $\mathrm{V}_{1}(0), \ldots, \mathrm{V}_{5}(0)$ at $n=0$ read,

$$
\begin{aligned}
& \mathrm{V}_{1}(0)=\mathrm{V}_{3}(0)= \\
& \frac{1}{\varepsilon} \frac{1}{8}+\frac{5}{4}+\varepsilon\left(8-\frac{21}{16} \zeta_{2}\right)+\varepsilon^{2}\left(42-\frac{23}{8} \zeta_{3}-\frac{105}{8} \zeta_{2}\right)+\varepsilon^{3}\left(198-\frac{115}{4} \zeta_{3}-84 \zeta_{2}+\frac{1017}{320} \zeta_{2}{ }^{2}\right) \\
& +\varepsilon^{4}\left(876-\frac{1053}{40} \zeta_{5}-184 \zeta_{3}-441 \zeta_{2}+\frac{483}{16} \zeta_{2} \zeta_{3}+\frac{1017}{32} \zeta_{2}^{2}\right)+\varepsilon^{5}\left(3728-\frac{1053}{4} \zeta_{5}\right. \\
& \left.-966 \zeta_{3}+\frac{529}{16} \zeta_{3}^{2}-2079 \zeta_{2}+\frac{2415}{8} \zeta_{2} \zeta_{3}+\frac{1017}{5} \zeta_{2}^{2}-\frac{24737}{4480} \zeta_{2}{ }^{3}\right), \\
& \mathrm{V}_{2}(0)= \\
& \frac{1}{\varepsilon} \frac{1}{8}+\frac{17}{16}+\varepsilon\left(\frac{183}{32}-\frac{17}{16} \zeta_{2}\right)+\varepsilon^{2}\left(\frac{1597}{64}-\frac{13}{8} \zeta_{3}-\frac{289}{32} \zeta_{2}\right)+\varepsilon^{3}\left(\frac{12359}{128}-\frac{221}{16} \zeta_{3}-\frac{3111}{64} \zeta_{2}\right. \\
& \left.+\frac{897}{320} \zeta_{2}^{2}\right)+\varepsilon^{4}\left(\frac{88629}{256}-\frac{303}{40} \zeta_{5}-\frac{2379}{32} \zeta_{3}-\frac{27149}{128} \zeta_{2}+\frac{221}{16} \zeta_{2} \zeta_{3}+\frac{15249}{640} \zeta_{2}^{2}\right) \\
& +\varepsilon^{5}\left(\frac{603871}{512}-\frac{5151}{80} \zeta_{5}-\frac{20761}{64} \zeta_{3}+\frac{169}{16} \zeta_{3}^{2}-\frac{210103}{256} \zeta_{2}+\frac{3757}{32} \zeta_{2} \zeta_{3}+\frac{164151}{1280} \zeta_{2}^{2}\right. \\
& \left.-\frac{12949}{4480} \zeta_{2}^{3}\right)
\end{aligned}
$$




$$
\begin{aligned}
\mathrm{V}_{4}(0) & = \\
& -\frac{1}{\varepsilon} \frac{1}{4}-\frac{11}{4}+\frac{1}{2} \zeta_{2}+\varepsilon\left(-\frac{77}{4}+\frac{5}{2} \zeta_{3}+\frac{49}{8} \zeta_{2}\right)+\varepsilon^{2}\left(-\frac{439}{4}+\frac{93}{4} \zeta_{3}+\frac{363}{8} \zeta_{2}-\frac{3}{4} \zeta_{2}^{2}\right) \\
& +\varepsilon^{3}\left(-\frac{229}{4}+\frac{75}{2} \zeta_{5}+\frac{583}{4} \zeta_{3}+\frac{2141}{8} \zeta_{2}-\frac{131}{4} \zeta_{2} \zeta_{3}-\frac{1857}{160} \zeta_{2}^{2}\right)+\varepsilon^{4}\left(-\frac{10527}{4}+\frac{6303}{20} \zeta_{5}\right. \\
& \left.+\frac{3081}{4} \zeta_{3}-45 \zeta_{3}^{2}+\frac{11111}{8} \zeta_{2}-\frac{2317}{8} \zeta_{2} \zeta_{3}-\frac{15147}{160} \zeta_{2}^{2}+\frac{421}{80} \zeta_{2}^{3}\right) \\
\mathrm{V}_{5}(0) & = \\
& \frac{1}{\varepsilon^{4}} \frac{5}{8}+\frac{1}{\varepsilon^{3}} \frac{5}{4}+\frac{1}{\varepsilon^{2}}\left(\frac{5}{2}-\frac{133}{16} \zeta_{2}\right)+\frac{1}{\varepsilon}\left(5-\frac{133}{8} \zeta_{2}-\frac{193}{8} \zeta_{3}\right)+10-\frac{133}{4} \zeta_{2}+\frac{5477}{320} \zeta_{2}^{2} \\
& -\frac{193}{4} \zeta_{3}+\varepsilon\left(20+\frac{4545}{16} \zeta_{2} \zeta_{3}-\frac{133}{2} \zeta_{2}+\frac{5477}{160} \zeta_{2}{ }^{2}-\frac{193}{2} \zeta_{3}-\frac{2303}{8} \zeta_{5}\right)+\varepsilon^{2}(40 \\
& \left.+\frac{4545}{8} \zeta_{2} \zeta_{3}-133 \zeta_{2}+\frac{5477}{80} \zeta_{2}{ }^{2}-\frac{578731}{13440} \zeta_{2}{ }^{3}-193 \zeta_{3}+\frac{5837}{16} \zeta_{3}^{2}-\frac{2303}{4} \zeta_{5}\right)
\end{aligned}
$$

which we have given in terms of the Riemann zeta-function consistently up to weight 6 . Previously, the result for $\mathrm{V}_{5}(0)$ has been obtained to weight 4 in Ref. [64].

Next we present the master integrals $\mathrm{R}_{1}(n), \ldots, \mathrm{R}_{6}(n)$ from the so-called real-real contributions. These masters can be defined through the following object:

$$
\begin{aligned}
\{i, \ldots, j\}= & \frac{e^{\left(3 \gamma_{\mathrm{e}} \varepsilon\right)}}{\pi^{3}} \int d^{d} p_{1} d^{d} p_{2} d^{d} p_{3} \delta\left(p_{1}^{2}\right) \delta\left(p_{2}^{2}\right) \delta\left(p_{3}^{2}\right) \delta\left(\left(q-p_{1}-p_{2}-p_{3}\right)^{2}\right) \\
& \times\left(2 q \cdot\left(q-p_{1}-p_{2}-p_{3}\right)\right)^{n} \frac{1}{Q_{i} \ldots Q_{j}},
\end{aligned}
$$

where $q \cdot q=1$ and the propagators are $Q_{1}=\left(q-p_{3}\right)^{2}, Q_{2}=\left(q-p_{2}\right)^{2}, Q_{3}=\left(q-p_{1}-p_{3}\right)^{2}, Q_{4}=$ $\left(q-p_{2}-p_{3}\right)^{2}, Q_{5}=\left(q-p_{1}-p_{2}\right)^{2}, Q_{6}=\left(p_{1}+p_{2}\right)^{2}, Q_{7}=\left(p_{1}+p_{3}\right)^{2}, Q_{8}=\left(p_{2}+p_{3}\right)^{2}$.

The $n$-dependent real-real masters are defined as: $\mathrm{R}_{1}(n)=\{-\}, \mathrm{R}_{2}(n)=\{1,2\}, \mathrm{R}_{3}(n)=$ $\{2,6\}, \mathrm{R}_{4}(n)=\{1,2,3,5\}, \mathrm{R}_{5}(n)=\{1,2,6,7\}, \mathrm{R}_{6}(n)=\{3,4,7,8\}$ and satisfy the following difference equations in $n$ together with boundary conditions at $n=0$ :

$$
\begin{aligned}
& (n+3-4 \varepsilon) \mathrm{R}_{1}(n)-(n+1-2 \varepsilon) \mathrm{R}_{1}(n-1)=0 \\
& (n+1-2 \varepsilon)(n+2-6 \varepsilon) \mathrm{R}_{2}(n)-(n-1)(n-4 \varepsilon) \mathrm{R}_{2}(n-2)= \\
& 2 \frac{(1-\varepsilon)(1-3 \varepsilon)(2-3 \varepsilon)(2 n+1-6 \varepsilon)}{(n-3 \varepsilon)(n+1-3 \varepsilon)} \mathrm{R}_{1}(n-2) \\
& (n+1-2 \varepsilon) \mathrm{R}_{3}(n)-n \mathrm{R}_{3}(n-1)=-\frac{(1-3 \varepsilon)(2-3 \varepsilon)(n+2-4 \varepsilon)}{n+1-3 \varepsilon} \frac{1}{\varepsilon} \mathrm{R}_{1}(n-1) \\
& (n-1-4 \varepsilon) \mathrm{R}_{4}(n)+(n-1-2 \varepsilon) \mathrm{R}_{4}(n-1)= \\
& 4 \frac{\mathrm{A}}{(n-2 \varepsilon)(n-3 \varepsilon)(n-4 \varepsilon)(n-1-3 \varepsilon)(n-1-4 \varepsilon)(n+1-3 \varepsilon)}\left(3240 \varepsilon^{6}-5058 n \varepsilon^{5}\right. \\
& -1098 \varepsilon^{5}+3282 n^{2} \varepsilon^{4}-279 \varepsilon^{4}+1407 n \varepsilon^{4}+52 \varepsilon^{3}+269 n \varepsilon^{3}-1135 n^{3} \varepsilon^{3}-707 n^{2} \varepsilon^{3} \\
& -41 n \varepsilon^{2}+174 n^{3} \varepsilon^{2}+5 \varepsilon^{2}+221 n^{4} \varepsilon^{2}-98 n^{2} \varepsilon^{2}+16 n^{3} \varepsilon-21 n^{4} \varepsilon+11 n^{2} \varepsilon-n \varepsilon-23 n^{5} \varepsilon
\end{aligned}
$$




$$
\begin{aligned}
& \left.-n^{4}-n^{3}+n^{5}+n^{6}\right) \frac{1}{\varepsilon^{2}} \mathrm{R}_{1}(n-1)+\frac{(n+1-2 \varepsilon)(n+2-6 \varepsilon)(n-6 \varepsilon)}{n-4 \varepsilon} \frac{1}{\varepsilon} \mathrm{R}_{2}(n) \\
& -\frac{(n-2 \varepsilon)(n+1-6 \varepsilon)(n-1-6 \varepsilon)}{n-1-4 \varepsilon} \frac{1}{\varepsilon} \mathrm{R}_{2}(n-1) \\
& (n-1-4 \varepsilon) \mathrm{R}_{5}(n)-(n-1-2 \varepsilon) \mathrm{R}_{5}(n-1)= \\
& 2 \frac{(1-3 \varepsilon)(2-3 \varepsilon)(n+2-4 \varepsilon)}{(n-3 \varepsilon)(n-4 \varepsilon)(n-1-4 \varepsilon)(n+1-3 \varepsilon)}\left(120 \varepsilon^{4}-154 n \varepsilon^{3}-142 \varepsilon^{3}+71 n^{2} \varepsilon^{2}\right. \\
& \left.+104 n \varepsilon^{2}+23 \varepsilon^{2}-10 n \varepsilon-25 n^{2} \varepsilon-14 n^{3} \varepsilon-\varepsilon+n^{2}+n^{4}+2 n^{3}\right) \frac{1}{\varepsilon^{2}} \mathrm{R}_{1}(n-1) \\
& +2(n-2 \varepsilon) \mathrm{R}_{3}(n-1)+\frac{(n+1-2 \varepsilon)(n+2-6 \varepsilon)(n-6 \varepsilon)}{\varepsilon} \frac{1}{\varepsilon} \mathrm{R}_{2}(n) \\
& +\frac{(n-2 \varepsilon)(n+1-6 \varepsilon)(n-1-6 \varepsilon)}{n-1-4 \varepsilon} \mathrm{R}_{2}(n-1), \\
& (n-1-4 \varepsilon) \mathrm{R}_{6}(n)-(n-1-2 \varepsilon) \mathrm{R}_{6}(n-1)=0 .
\end{aligned}
$$

$\mathrm{R}_{1}(0)=$

$$
\begin{aligned}
& \frac{1}{96}+\varepsilon \frac{71}{576}+\varepsilon^{2}\left(\frac{3115}{3456}-\frac{7}{64} \zeta_{2}\right)+\varepsilon^{3}\left(\frac{109403}{20736}-\frac{29}{96} \zeta_{3}-\frac{497}{384} \zeta_{2}\right)+\varepsilon^{4}\left(\frac{3386467}{124416}-\frac{2059}{576} \zeta_{3}\right. \\
& \left.-\frac{21805}{2304} \zeta_{2}+\frac{291}{1280} \zeta_{2}{ }^{2}\right)+\varepsilon^{5}\left(\frac{96885467}{746496}-\frac{421}{160} \zeta_{5}-\frac{90335}{3456} \zeta_{3}-\frac{765821}{13824} \zeta_{2}+\frac{203}{64} \zeta_{2} \zeta_{3}\right.
\end{aligned}
$$$$
\left.+\frac{6887}{2560} \zeta_{2}{ }^{2}\right)+\varepsilon^{6}\left(\frac{2631913075}{4478976}-\frac{29891}{960} \zeta_{5}-\frac{3172687}{20736} \zeta_{3}+\frac{841}{192} \zeta_{3}{ }^{2}-\frac{23705269}{82944} \zeta_{2}\right.
$$$$
\left.+\frac{14413}{384} \zeta_{2} \zeta_{3}+\frac{60431}{3072} \zeta_{2}^{2}-\frac{15089}{53760} \zeta_{2}^{3}\right)
$$

$\mathrm{R}_{2}(0)=$

$-\frac{1}{8}+\frac{1}{8} \zeta_{2}+\varepsilon\left(-\frac{7}{4}+\frac{9}{8} \zeta_{3}+\frac{7}{8} \zeta_{2}\right)+\varepsilon^{2}\left(-\frac{119}{8}+\frac{63}{8} \zeta_{3}+\frac{87}{16} \zeta_{2}+\frac{97}{80} \zeta_{2}{ }^{2}\right)+\varepsilon^{3}\left(-\frac{199}{2}\right.$

$\left.+\frac{207}{8} \zeta_{5}+\frac{163}{4} \zeta_{3}+\frac{139}{4} \zeta_{2}-\frac{211}{16} \zeta_{2} \zeta_{3}+\frac{679}{80} \zeta_{2}^{2}\right)+\varepsilon^{4}\left(-\frac{4617}{8}+\frac{1449}{8} \zeta_{5}+\frac{1585}{8} \zeta_{3}\right.$

$\left.-\frac{45}{2} \zeta_{3}^{2}+\frac{3445}{16} \zeta_{2}-\frac{1477}{16} \zeta_{2} \zeta_{3}+\frac{11931}{320} \zeta_{2}^{2}+\frac{1141}{320} \zeta_{2}{ }^{3}\right)$

$\mathrm{R}_{3}(0)=$

$-\frac{1}{\varepsilon} \frac{1}{8}-\frac{11}{8}+\varepsilon\left(-\frac{77}{8}+\frac{21}{16} \zeta_{2}\right)+\varepsilon^{2}\left(-\frac{439}{8}+\frac{29}{8} \zeta_{3}+\frac{231}{16} \zeta_{2}\right)+\varepsilon^{3}\left(-\frac{2229}{8}+\frac{319}{8} \zeta_{3}\right.$

$\left.+\frac{1617}{16} \zeta_{2}-\frac{873}{320} \zeta_{2}{ }^{2}\right)+\varepsilon^{4}\left(-\frac{10527}{8}+\frac{1263}{40} \zeta_{5}+\frac{2233}{8} \zeta_{3}+\frac{9219}{16} \zeta_{2}-\frac{609}{16} \zeta_{2} \zeta_{3}\right.$

$\left.-\frac{9603}{320} \zeta_{2}^{2}\right)+\varepsilon^{5}\left(-\frac{47389}{8}+\frac{13893}{40} \zeta_{5}+\frac{12731}{8} \zeta_{3}-\frac{841}{16} \zeta_{3}{ }^{2}+\frac{46809}{16} \zeta_{2}-\frac{6699}{16} \zeta_{2} \zeta_{3}\right.$

$\left.-\frac{67221}{320} \zeta_{2}^{2}+\frac{15089}{4480} \zeta_{2}^{3}\right)$ 


$$
\begin{aligned}
\mathrm{R}_{4}(0) & =\mathrm{R}_{5}(0)= \\
& \frac{1}{\varepsilon^{4}} \frac{3}{32}+\frac{1}{\varepsilon^{3}} \frac{3}{16}+\frac{1}{\varepsilon^{2}}\left(\frac{3}{8}-\frac{83}{64} \zeta_{2}\right)+\frac{1}{\varepsilon}\left(\frac{3}{4}-\frac{185}{32} \zeta_{3}-\frac{83}{32} \zeta_{2}\right)+\frac{3}{2}-\frac{185}{16} \zeta_{3}-\frac{83}{16} \zeta_{2}-\frac{457}{256} \zeta_{2}^{2} \\
+ & \varepsilon\left(3-\frac{15899}{160} \zeta_{5}-\frac{185}{8} \zeta_{3}-\frac{83}{8} \zeta_{2}+\frac{4137}{64} \zeta_{2} \zeta_{3}-\frac{457}{128} \zeta_{2}^{2}\right)+\varepsilon^{2}\left(6-\frac{15899}{80} \zeta_{5}-\frac{185}{4} \zeta_{3}\right. \\
& \left.+\frac{6667}{64} \zeta_{3}^{2}-\frac{83}{4} \zeta_{2}+\frac{4137}{32} \zeta_{2} \zeta_{3}-\frac{457}{64} \zeta_{2}^{2}-\frac{692333}{53760} \zeta_{2}^{3}\right) \\
\mathrm{R}_{6}(0) & = \\
& \frac{1}{\varepsilon^{4}} \frac{5}{8}+\frac{1}{\varepsilon^{3}} \frac{5}{4}+\frac{1}{\varepsilon^{2}}\left(\frac{5}{2}-\frac{105}{16} \zeta_{2}\right)+\frac{1}{\varepsilon}\left(5-\frac{141}{8} \zeta_{3}-\frac{105}{8} \zeta_{2}\right)+10-\frac{141}{4} \zeta_{3}-\frac{105}{4} \zeta_{2} \\
& +\frac{969}{64} \zeta_{2}{ }^{2}+\varepsilon\left(20-\frac{1119}{8} \zeta_{5}-\frac{141}{2} \zeta_{3}-\frac{105}{2} \zeta_{2}+\frac{2961}{16} \zeta_{2} \zeta_{3}+\frac{969}{32} \zeta_{2}^{2}\right)+\varepsilon^{2}\left(40-\frac{1119}{4} \zeta_{5}\right. \\
& \left.-141 \zeta_{3}+\frac{4005}{16} \zeta_{3}^{2}-105 \zeta_{2}+\frac{2961}{8} \zeta_{2} \zeta_{3}+\frac{969}{16} \zeta_{2}^{2}-\frac{15121}{896} \zeta_{2}{ }^{3}\right) .
\end{aligned}
$$

Again we have given the results consistently up to weight 6 in the Riemann zeta-function. The difference equation for $\mathrm{R}_{2}(n)$ which is of second order needs actually two boundary conditions. The value for $\mathrm{R}_{2}(1)$ is obtained from an additional fixed- $n$ reduction

$$
\mathrm{R}_{2}(1)=\frac{3-4 \varepsilon}{1-2 \varepsilon} \mathrm{R}_{1}(0)
$$

The values for $\mathrm{R}_{4}(0), \mathrm{R}_{5}(0)$ and $\mathrm{R}_{6}(0)$ have been obtained before up to weight 4 in Ref. [64].

\section{Appendix B: The exact Mellin-space results}

Here we give the exact Mellin- $N$ expressions for the time-like splitting functions and the coefficient functions $c_{T}, c_{L}$ and $c_{A}$ up to second order in $a_{\mathrm{s}}=\alpha_{\mathrm{s}} /(4 \pi)$, expressed in terms of harmonic sums, recursively defined by [61]

$$
S_{ \pm m_{1}, m_{2}, \ldots, m_{k}}(N)=\sum_{i=1}^{N} \frac{( \pm 1)^{i}}{i^{m_{1}}} S_{m_{2}, \ldots, m_{k}}(i), \quad S(N)=1
$$

and we employ the notation

$$
\mathbf{N}_{ \pm} S_{\vec{m}}=S_{\vec{m}}(N \pm 1), \quad \mathbf{N}_{ \pm \mathbf{i}} S_{\vec{m}}=S_{\vec{m}}(N \pm i)
$$

The well known results for the LO anomalous dimensions (cf. Eq. (25) in $N$-space are:

$$
\begin{aligned}
& \gamma_{\mathrm{qq}}^{(0)}(N)=C_{F}\left\{-3+\left(2 \mathbf{N}_{-}+2 \mathbf{N}_{+}\right) S_{1}\right\}, \\
& \gamma_{\mathrm{qg}}^{(0)}(N)=n_{f}\left\{2 \mathbf{N}_{-}+8 \mathbf{N}_{+}-4 \mathbf{N}_{+2}-6\right\} S_{1},
\end{aligned}
$$




$$
\begin{aligned}
& \gamma_{\mathrm{gq}}^{(0)}(N)=C_{F}\left\{-8 \mathbf{N}_{-}+4 \mathbf{N}_{-\mathbf{2}}-2 \mathbf{N}_{+}+6\right\} S_{1}, \\
& \gamma_{\mathrm{gg}}^{(0)}(N)=C_{A}\left\{-\frac{11}{3}+\left(-8 \mathbf{N}_{-}+4 \mathbf{N}_{-\mathbf{2}}-8 \mathbf{N}_{+}+4 \mathbf{N}_{+\mathbf{2}}+12\right) S_{1}\right\}+\frac{2}{3} n_{f} .
\end{aligned}
$$

For the NLO anomalous dimensions (cf. Eq. (25) a distinction between even and odd Mellin moments is in order. We give the respective expressions for the even Mellin moments of $\gamma_{\mathrm{ns}}^{(1)+}$ in Eq. (B.7) and $\gamma_{\mathrm{qq}}^{(1) \mathrm{s}}, \gamma_{\mathrm{qg}}^{(1)}, \gamma_{\mathrm{gq}}^{(1)}, \gamma_{\mathrm{gg}}^{(1)}$ in Eqs. (B.9)-(B.12) and for the odd Mellin moments of $\gamma_{\mathrm{ns}}^{(1)-}$ in Eq. (B.8). Note also, that we do not obtain $\gamma_{\mathrm{gg}}^{(1)}$ and $\gamma_{\mathrm{gg}}^{(1)}$ since we only consider vector boson decays (see discussion in Section 4). Nevertheless, we also quote these quantities here for completeness.

$$
\begin{aligned}
& \gamma_{\mathrm{ns}}^{(1)+}(N)= \\
& C_{F}^{2}\left\{\left(8-\left(16 \zeta_{2}+4\right) \mathbf{N}_{+}-\left(16 \zeta_{2}+4\right) \mathbf{N}_{-}\right) S_{1}-\left(4 \mathbf{N}_{+}+8\right) S_{2}-\left(4 \mathbf{N}_{-}+28 \mathbf{N}_{+}\right) S_{3}+\right. \\
& \left.\left(-16 \mathbf{N}_{-}-16 \mathbf{N}_{+}\right) S_{1,-2}+\left(8 \mathbf{N}_{-}+8 \mathbf{N}_{+}\right) S_{1,2}+\left(8 \mathbf{N}_{-}+8 \mathbf{N}_{+}\right) S_{2,1}+16 S_{-3}+24 \zeta_{2}-\frac{3}{2}\right\}+ \\
& C_{F} C_{A}\left\{-8 S_{-3}+\left(\frac{302}{9} \mathbf{N}_{+}-\frac{112}{3}+\frac{302}{9} \mathbf{N}_{-}\right) S_{1}+\left(-\frac{22}{3} \mathbf{N}_{-}-\frac{22}{3} \mathbf{N}_{+}\right) S_{2}+8 \mathbf{N}_{+} S_{3}-\right. \\
& \left.\frac{17}{6}+\left(8 \mathbf{N}_{-}+8 \mathbf{N}_{+}\right) S_{1,-2}\right\}+ \\
& C_{F} n_{f}\left\{\left(-\frac{44}{9} \mathbf{N}_{+}-\frac{44}{9} \mathbf{N}_{-}+\frac{16}{3}\right) S_{1}+\frac{1}{3}+\left(\frac{4}{3} \mathbf{N}_{-}+\frac{4}{3} \mathbf{N}_{+}\right) S_{2}\right\}, \\
& \gamma_{\mathrm{ns}}^{(1)-}(N)= \\
& C_{F}^{2}\left\{16 S_{-3}-\left(\left(16 \zeta_{2}+36\right) \mathbf{N}_{+}-72+\left(16 \zeta_{2}+36\right) \mathbf{N}_{-}\right) S_{1}+\left(16 \mathbf{N}_{-}-20 \mathbf{N}_{+}-8\right) S_{2}-\right. \\
& \left(20 \mathbf{N}_{-}+12 \mathbf{N}_{+}\right) S_{3}+\left(8 \mathbf{N}_{+}+8 \mathbf{N}_{-}\right) S_{2,1}-\left(16 \mathbf{N}_{-}+16 \mathbf{N}_{+}\right) S_{1,-2}+\left(8 \mathbf{N}_{+}+8 \mathbf{N}_{-}\right) S_{1,2}- \\
& \left.\frac{3}{2}+24 \zeta_{2}\right\}+ \\
& C_{F} C_{A}\left\{-8 S_{-3}+\left(\frac{446}{9} \mathbf{N}_{-}-\frac{208}{3}+\frac{446}{9} \mathbf{N}_{+}\right) S_{1}+\left(-\frac{46}{3} \mathbf{N}_{-}+\frac{2}{3} \mathbf{N}_{+}\right) S_{2}+8 \mathbf{N}_{-} S_{3}-\right. \\
& \left.\frac{17}{6}+\left(8 \mathbf{N}_{+}+8 \mathbf{N}_{-}\right) S_{1,-2}\right\}+ \\
& C_{F} n_{f}\left\{\left(-\frac{44}{9} \mathbf{N}_{+}-\frac{44}{9} \mathbf{N}_{-}+\frac{16}{3}\right) S_{1}+\frac{1}{3}+\left(\frac{4}{3} \mathbf{N}_{+}+\frac{4}{3} \mathbf{N}_{-}\right) S_{2}\right\}, \\
& \gamma_{\mathrm{qq}}^{(1) \mathrm{s}}(N)= \\
& C_{F} n_{f}\left\{\left(-\frac{208}{9} \mathbf{N}_{-}-\frac{224}{9} \mathbf{N}_{+\mathbf{2}}+48-\frac{80}{9} \mathbf{N}_{-\mathbf{2}}+\frac{80}{9} \mathbf{N}_{+}\right) S_{1}+\right. \\
& \left.\left(-\frac{76}{3} \mathbf{N}_{+}-\frac{32}{3} \mathbf{N}_{+\mathbf{2}}+16+20 \mathbf{N}_{-}\right) S_{2}+\left(8 \mathbf{N}_{-}-8 \mathbf{N}_{+}\right) S_{3}\right\} \text {, }
\end{aligned}
$$




$$
\begin{aligned}
& \gamma_{\mathrm{qg}}^{(1)}(N)= \\
& C_{F} n_{f}\left\{\left(-86 \mathbf{N}_{+}+40 \mathbf{N}_{+\mathbf{2}}+70-24 \mathbf{N}_{-}\right) S_{1}+\left(10 \mathbf{N}_{-}+8 \mathbf{N}_{+\mathbf{2}}-18\right) S_{2}+\left(16 \mathbf{N}_{+\mathbf{2}}+12-\right.\right. \\
& \left.4 \mathbf{N}_{-}-24 \mathbf{N}_{+}\right) S_{3}+\left(16 \mathbf{N}_{+}-20-8 \mathbf{N}_{+2}+12 \mathbf{N}_{-}\right) S_{1,1}+\left(96 \mathbf{N}_{+}+24 \mathbf{N}_{-}-48 \mathbf{N}_{+2}-\right. \\
& \left.72) S_{1,2}+\left(24-8 \mathbf{N}_{-}+16 \mathbf{N}_{+\mathbf{2}}-32 \mathbf{N}_{+}\right) S_{2,1}+\left(24-8 \mathbf{N}_{-}+16 \mathbf{N}_{+\mathbf{2}}-32 \mathbf{N}_{+}\right) S_{1,1,1}\right\}+ \\
& C_{A} n_{f}\left\{\left(\left(\frac{44}{3}-16 \zeta_{2}\right) \mathbf{N}_{-}-\left(64 \zeta_{2}+\frac{1564}{9}\right) \mathbf{N}_{+}+\left(32 \zeta_{2}-\frac{712}{9}\right) \mathbf{N}_{+2}+48 \zeta_{2}-\right.\right. \\
& \left.\frac{80}{9} \mathbf{N}_{-2}+\frac{2224}{9}\right) S_{1}+\left(-\frac{16}{3} \mathbf{N}_{+2}+40-40 \mathbf{N}_{+}+\frac{16}{3} \mathbf{N}_{-}\right) S_{2}+\left(8 \mathbf{N}_{-}-48 \mathbf{N}_{+}+40\right) S_{3}+ \\
& \left(24-8 \mathbf{N}_{-}+16 \mathbf{N}_{+\mathbf{2}}-32 \mathbf{N}_{+}\right) S_{1,-2}+\left(-\frac{80}{3} \mathbf{N}_{+}+\frac{40}{3} \mathbf{N}_{+2}+28-\frac{44}{3} \mathbf{N}_{-}\right) S_{1,1}+ \\
& \left(48-16 \mathbf{N}_{-}-64 \mathbf{N}_{+}+32 \mathbf{N}_{+2}\right) S_{1,2}+\left(64 \mathbf{N}_{+}+16 \mathbf{N}_{-}-48-32 \mathbf{N}_{+2}\right) S_{2,1}+ \\
& \left.\left(8 \mathbf{N}_{-}+32 \mathbf{N}_{+}-16 \mathbf{N}_{+2}-24\right) S_{1,1,1}\right\}+ \\
& n_{f}^{2}\left\{\left(8-\frac{40}{9} \mathbf{N}_{-}+\frac{32}{9} \mathbf{N}_{+2}-\frac{64}{9} \mathbf{N}_{+}\right) S_{1}+\left(-8+\frac{8}{3} \mathbf{N}_{-}-\frac{16}{3} \mathbf{N}_{+\mathbf{2}}+\frac{32}{3} \mathbf{N}_{+}\right) S_{2}+\right. \\
& \left.\left(-8+\frac{8}{3} \mathbf{N}_{-}-\frac{16}{3} \mathbf{N}_{+2}+\frac{32}{3} \mathbf{N}_{+}\right) S_{1,1}\right\} \\
& \gamma_{\mathrm{gq}}^{(1)}(N)= \\
& C_{F}^{2}\left\{\left(\left(16 \zeta_{2}-18\right) \mathbf{N}_{+}+\left(64 \zeta_{2}-2\right) \mathbf{N}_{-}-48 \zeta_{2}+20-32 \zeta_{2} \mathbf{N}_{-2}\right) S_{1}+\left(32 \mathbf{N}_{-}-34+\right.\right. \\
& \left.2 \mathbf{N}_{+}\right) S_{2}+\left(8 \mathbf{N}_{-}-12+4 \mathbf{N}_{+}\right) S_{3}+\left(8 \mathbf{N}_{+}-8\right) S_{1,1}+\left(16 \mathbf{N}_{+}-32 \mathbf{N}_{-2}+64 \mathbf{N}_{-}-\right. \\
& \left.48) S_{1,2}+\left(48-64 \mathbf{N}_{-}+32 \mathbf{N}_{-2}-16 \mathbf{N}_{+}\right) S_{2,1}+\left(24-8 \mathbf{N}_{+}+16 \mathbf{N}_{-2}-32 \mathbf{N}_{-}\right) S_{1,1,1}\right\}+ \\
& C_{F} C_{A}\left\{\left(\frac{68}{9} \mathbf{N}_{-\mathbf{2}}+\frac{176}{9} \mathbf{N}_{+\mathbf{2}}+\frac{112}{9} \mathbf{N}_{-}-24-\frac{140}{9} \mathbf{N}_{+}\right) S_{1}+\left(\frac{32}{3} \mathbf{N}_{+\mathbf{2}}+\frac{76}{3} \mathbf{N}_{+}+24 \mathbf{N}_{-\mathbf{2}}-\right.\right. \\
& \left.4-56 \mathbf{N}_{-}\right) S_{2}+\left(24 \mathbf{N}_{+}+16 \mathbf{N}_{-}-8-32 \mathbf{N}_{-2}\right) S_{3}+\left(32 \mathbf{N}_{-}-16 \mathbf{N}_{-2}+8 \mathbf{N}_{+}-24\right) S_{1,-2}+ \\
& \left(8-8 \mathbf{N}_{+}\right) S_{1,1}+\left(48 \mathbf{N}_{-2}-24 \mathbf{N}_{+}+72-96 \mathbf{N}_{-}\right) S_{1,2}+\left(32 \mathbf{N}_{-}-16 \mathbf{N}_{-2}+8 \mathbf{N}_{+}-24\right) S_{2,1}+ \\
& \left.\left(-24+32 \mathbf{N}_{-}-16 \mathbf{N}_{-2}+8 \mathbf{N}_{+}\right) S_{1,1,1}\right\} \\
& \gamma_{\mathrm{gg}}^{(1)}(N)= \\
& C_{F} n_{f}\left\{\left(32+\frac{328}{9} \mathbf{N}_{+\mathbf{2}}-\frac{256}{9} \mathbf{N}_{-}+\frac{184}{9} \mathbf{N}_{-2}-\frac{544}{9} \mathbf{N}_{+}\right) S_{1}+\right.
\end{aligned}
$$




$$
\begin{aligned}
& \left.\left(\frac{32}{3} \mathbf{N}_{+\mathbf{2}}-8-\frac{28}{3} \mathbf{N}_{-}-\frac{32}{3} \mathbf{N}_{-\mathbf{2}}+\frac{52}{3} \mathbf{N}_{+}\right) S_{2}+2+\left(8 \mathbf{N}_{-}-8 \mathbf{N}_{+}\right) S_{3}\right\}+ \\
& C_{A}^{2}\left\{8 S_{-3}+\left(\left(\frac{218}{9}+64 \zeta_{2}\right) \mathbf{N}_{+}-32 \zeta_{2} \mathbf{N}_{+2}+\left(\frac{218}{9}+64 \zeta_{2}\right) \mathbf{N}_{-}-96 \zeta_{2}-\frac{56}{3}-\right.\right. \\
& \left.32 \zeta_{2} \mathbf{N}_{-\mathbf{2}}\right) S_{1}+\left(-\frac{88}{3} \mathbf{N}_{+\mathbf{2}}-40+\frac{88}{3} \mathbf{N}_{-2}+\frac{76}{3} \mathbf{N}_{+}-\frac{44}{3} \mathbf{N}_{-}\right) S_{2}+\left(-16 \mathbf{N}_{+\mathbf{2}}-\right. \\
& \left.32 \mathbf{N}_{-\mathbf{2}}-72+16 \mathbf{N}_{-}+80 \mathbf{N}_{+}\right) S_{3}+\left(32 \mathbf{N}_{+}+32 \mathbf{N}_{-}-16 \mathbf{N}_{+\mathbf{2}}-16 \mathbf{N}_{-\mathbf{2}}-48\right) S_{1,-2}+ \\
& \left(48-32 \mathbf{N}_{+}+16 \mathbf{N}_{+\mathbf{2}}-32 \mathbf{N}_{-}+16 \mathbf{N}_{-2}\right) S_{1,2}+ \\
& \left.\left(48-32 \mathbf{N}_{+}+16 \mathbf{N}_{+\mathbf{2}}-32 \mathbf{N}_{-}+16 \mathbf{N}_{-\mathbf{2}}\right) S_{2,1}+\frac{88}{3} \zeta_{2}-\frac{32}{3}\right\}+ \\
& C_{A} n_{f}\left\{\left(\frac{56}{3} \mathbf{N}_{-}-\frac{92}{9} \mathbf{N}_{+\mathbf{2}}-\frac{64}{3}-\frac{92}{9} \mathbf{N}_{-\mathbf{2}}+\frac{56}{3} \mathbf{N}_{+}\right) S_{1}+\right. \\
& \left.\left(-8 \mathbf{N}_{-}+\frac{16}{3} \mathbf{N}_{-\mathbf{2}}+\frac{16}{3} \mathbf{N}_{+\mathbf{2}}-\frac{40}{3} \mathbf{N}_{+}+16\right) S_{2}+\frac{8}{3}-\frac{16}{3} \zeta_{2}\right\}
\end{aligned}
$$

The $O\left(\alpha_{\mathrm{s}}\right)$ coefficient functions in $N$-space read:

$$
\begin{aligned}
& c_{T, \mathrm{q}}^{(1)}(N)=C_{F}\left\{\left(-3 \mathbf{N}_{+}+6\right) S_{1}+\left(4 \mathbf{N}_{-}+4 \mathbf{N}_{+}\right) S_{2}-9+\left(2 \mathbf{N}_{-}+2 \mathbf{N}_{+}\right) S_{1,1}\right\}, \\
& c_{L, \mathrm{q}}^{(1)}(N)=C_{F}\left(2-2 \mathbf{N}_{-}\right) S_{1}, \\
& c_{A, \mathrm{q}}^{(1)}(N)-c_{T, \mathrm{q}}^{(1)}(N)=C_{F}\left(2 \mathbf{N}_{-}+2 \mathbf{N}_{+}-4\right) S_{1}, \\
& c_{T, \mathrm{~g}}^{(1)}(N)=C_{F}\left\{\left(-16 \mathbf{N}_{-}+8 \mathbf{N}_{-2}+8\right) S_{1}+\left(16 \mathbf{N}_{-2}-8 \mathbf{N}_{+}+24-32 \mathbf{N}_{-}\right) S_{2}\right. \\
& \left.+\left(8 \mathbf{N}_{-2}-4 \mathbf{N}_{+}+12-16 \mathbf{N}_{-}\right) S_{1,1}\right\}, \\
& c_{L, \mathrm{~g}}^{(1)}(N)=C_{F}\left(16 \mathbf{N}_{-}-8 \mathbf{N}_{-2}-8\right) S_{1} .
\end{aligned}
$$

Finally, for the $O\left(\alpha_{\mathrm{s}}^{2}\right)$ coefficient functions we give the even Mellin moments of $c_{I, \mathrm{~ns}}^{(2)}, c_{I, \mathrm{ps}}^{(2)}, c_{I, \mathrm{~g}}^{(2)}$ with $I=T, L$ in Eqs. B.18- B.23 and the odd Mellin moments of $c_{A, \mathrm{~ns}}^{(2)}$ in Eq. (B.24).

$$
\begin{aligned}
& c_{T, \mathrm{~ns}}^{(2)}(N)= \\
& \quad \delta(N-2)\left[C_{F}^{2}\left\{-\frac{560}{9} \zeta_{2}-\frac{704}{15} \zeta_{3}+\frac{145517}{810}\right\}+\right. \\
& \left.\quad C_{F} C_{A}\left\{-\frac{52}{9} \zeta_{2}-\frac{316}{5} \zeta_{3}+\frac{127349}{810}\right\}+C_{F} n_{f}\left\{-\frac{2354}{81}+8 \zeta_{3}+\frac{16}{9} \zeta_{2}\right\}\right]+ \\
& \quad \theta(N-3)\left[C _ { F } { } ^ { 2 } \left\{59 \zeta_{2}-12 \zeta_{3}+72 S_{-4}-16 S_{-2}+16 S_{-3,1}+48 S_{-2,-2}+\frac{331}{8}+\left(\left(-\frac{118}{5}+\right.\right.\right.\right.
\end{aligned}
$$




$$
\begin{aligned}
& \left.\left.12 \zeta_{2}\right) \mathbf{N}_{+}-\frac{48}{5} \mathbf{N}_{+2}+\left(4 \zeta_{2}-\frac{282}{5}\right) \mathbf{N}_{-}-26+\frac{48}{5} \mathbf{N}_{-2}\right) S_{2}+\left(\left(8 \zeta_{3}-\frac{75}{2}-12 \zeta_{2}\right) \mathbf{N}_{+}+\right. \\
& \left.\frac{48}{5} \mathbf{N}_{+\mathbf{2}}+\left(-63-12 \zeta_{2}+8 \zeta_{3}\right) \mathbf{N}_{-}-48 \zeta_{3}+\frac{48}{5} \mathbf{N}_{-\mathbf{2}}+\frac{279}{5}\right) S_{1}+\left(\frac{48}{5} \mathbf{N}_{+\mathbf{3}}-36-\frac{48}{5} \mathbf{N}_{+\mathbf{2}}-\right. \\
& \left.30 \mathbf{N}_{+}\right) S_{3}-\left(22 \mathbf{N}_{-}+138 \mathbf{N}_{+}\right) S_{4}-\left(16 \mathbf{N}_{-}+16 \mathbf{N}_{+}\right) S_{1,-2,1}+\left(32-48 \mathbf{N}_{-}-48 \mathbf{N}_{+}\right) S_{1,1,-2}+ \\
& \left(-16-16 \mathbf{N}_{-}-16 \mathbf{N}_{+}\right) S_{1,-3}+\left(-8 \mathbf{N}_{-}-\frac{48}{5} \mathbf{N}_{-\mathbf{2}}+16-\frac{48}{5} \mathbf{N}_{+\mathbf{2}}+\frac{48}{5} \mathbf{N}_{+\mathbf{3}}+\frac{48}{5} \mathbf{N}_{-\mathbf{3}}-\right. \\
& \left.8 \mathbf{N}_{+}\right) S_{1,-2}+\left(56-28 \mathbf{N}_{+}-4 \mathbf{N}_{-}\right) S_{1,2}+\left(64-\left(32 \zeta_{2}+51\right) \mathbf{N}_{+}-\left(32 \zeta_{2}+40\right) \mathbf{N}_{-}\right) S_{1,1}+ \\
& \left(-28 \mathbf{N}_{-}+16-28 \mathbf{N}_{+}\right) S_{1,3}+\left(-16-32 \mathbf{N}_{+}+32 \mathbf{N}_{-}\right) S_{2,-2}+\left(-16+24 \mathbf{N}_{-}+4 \mathbf{N}_{+}\right) S_{2,1}+ \\
& \left(36 \mathbf{N}_{-}+36 \mathbf{N}_{+}\right) S_{2,2}+\left(16 \mathbf{N}_{-}-24 \mathbf{N}_{+}\right) S_{3,1}+\left(8+20 \mathbf{N}_{-}+8 \mathbf{N}_{+}\right) S_{1,1,1}+\left(24 \mathbf{N}_{-}+\right. \\
& \left.\left.24 \mathbf{N}_{+}\right) S_{1,1,2}+\left(16 \mathbf{N}_{+}+16 \mathbf{N}_{-}\right) S_{1,2,1}+\left(16 \mathbf{N}_{+}+24 \mathbf{N}_{-}\right) S_{2,1,1}+\left(24 \mathbf{N}_{-}+24 \mathbf{N}_{+}\right) S_{1,1,1,1}\right\}+ \\
& C_{F} C_{A}\left\{8 S_{-2}+\left(\left(\frac{1225}{54}-36 \zeta_{3}-\frac{22}{3} \zeta_{2}\right) \mathbf{N}_{+}-\frac{24}{5} \mathbf{N}_{+2}+\left(\frac{1580}{27}-36 \zeta_{3}-\frac{22}{3} \zeta_{2}\right) \mathbf{N}_{-}+\right.\right. \\
& \left.24 \zeta_{3}-\frac{24}{5} \mathbf{N}_{-2}-\frac{593}{45}\right) S_{1}+\left(\left(\frac{119}{5}-8 \zeta_{2}\right) \mathbf{N}_{+}+\frac{24}{5} \mathbf{N}_{+2}+\left(\frac{863}{15}-8 \zeta_{2}\right) \mathbf{N}_{-}-\frac{24}{5} \mathbf{N}_{-2}-\right. \\
& \left.\frac{38}{3}\right) S_{2}+\left(\frac{23}{3} \mathbf{N}_{+}+\frac{24}{5} \mathbf{N}_{+2}-\frac{24}{5} \mathbf{N}_{+3}-4+\frac{11}{3} \mathbf{N}_{-}\right) S_{3}+36 \mathbf{N}_{+} S_{4}-8 S_{-3,1}-24 S_{-2,-2}+ \\
& \left(8+8 \mathbf{N}_{-}+8 \mathbf{N}_{+}\right) S_{1,-3}+\left(4 \mathbf{N}_{+}-\frac{24}{5} \mathbf{N}_{-\mathbf{3}}-\frac{24}{5} \mathbf{N}_{+\mathbf{3}}-8+\frac{24}{5} \mathbf{N}_{+\mathbf{2}}+\frac{24}{5} \mathbf{N}_{-\mathbf{2}}+\right. \\
& \left.4 \mathbf{N}_{-}\right) S_{1,-2}+\left(\left(\frac{311}{9}+8 \zeta_{2}\right) \mathbf{N}_{+}-\frac{112}{3}+\left(\frac{392}{9}+8 \zeta_{2}\right) \mathbf{N}_{-}\right) S_{1,1}+\left(12 \mathbf{N}_{-}+12 \mathbf{N}_{+}-\right. \\
& 8) S_{1,3}+\left(8-16 \mathbf{N}_{-}+16 \mathbf{N}_{+}\right) S_{2,-2}+\left(\frac{22}{3} \mathbf{N}_{-}+\frac{22}{3} \mathbf{N}_{+}\right) S_{2,1}+\left(4 \mathbf{N}_{-}+4 \mathbf{N}_{+}\right) S_{2,2}+ \\
& \left(-4 \mathbf{N}_{-}+4 \mathbf{N}_{+}\right) S_{3,1}+\left(8 \mathbf{N}_{-}+8 \mathbf{N}_{+}\right) S_{1,-2,1}+\left(-16+24 \mathbf{N}_{+}+24 \mathbf{N}_{-}\right) S_{1,1,-2}+\left(\frac{22}{3} \mathbf{N}_{-}+\right. \\
& \left.\left.\frac{22}{3} \mathbf{N}_{+}\right) S_{1,1,1}-\left(4 \mathbf{N}_{-}+4 \mathbf{N}_{+}\right) S_{1,1,2}+\left(4 \mathbf{N}_{-}+4 \mathbf{N}_{+}\right) S_{1,2,1}-36 S_{-4}+7 \zeta_{2}+10 \zeta_{3}-\frac{5465}{72}\right\}+ \\
& C_{F} n_{f}\left\{\left(\left(\frac{4}{3} \zeta_{2}-\frac{17}{27}\right) \mathbf{N}_{+}-\frac{14}{9}+\left(-\frac{188}{27}+\frac{4}{3} \zeta_{2}\right) \mathbf{N}_{-}\right) S_{1}+\left(\frac{8}{3}-\frac{22}{3} \mathbf{N}_{-}-6 \mathbf{N}_{+}\right) S_{2}+\right. \\
& \left(-\frac{2}{3} \mathbf{N}_{+}-\frac{2}{3} \mathbf{N}_{-}\right) S_{3}+\left(-\frac{26}{9} \mathbf{N}_{+}-\frac{44}{9} \mathbf{N}_{-}+\frac{4}{3}\right) S_{1,1}+\left(-\frac{4}{3} \mathbf{N}_{+}-\frac{4}{3} \mathbf{N}_{-}\right) S_{2,1}+ \\
& \left.\left.\left(-\frac{4}{3} \mathbf{N}_{+}-\frac{4}{3} \mathbf{N}_{-}\right) S_{1,1,1}-2 \zeta_{2}+\frac{457}{36}+8 \zeta_{3}\right\}\right] \text {, } \\
& c_{T, \mathrm{ps}}^{(2)}(N)= \\
& \delta(N-2) C_{F} n_{f}\left[\frac{2462}{81}-\frac{16}{9} \zeta_{2}\right]+
\end{aligned}
$$




$$
\begin{aligned}
& \theta(N-3) C_{F} n_{f}\left[\left(\left(\frac{118}{27}-\frac{4}{3} \zeta_{2}\right) \mathbf{N}_{+}+\left(\frac{512}{27}+\frac{16}{3} \zeta_{2}\right) \mathbf{N}_{+2}+\left(\frac{982}{27}-\frac{4}{3} \zeta_{2}\right) \mathbf{N}_{-}+\left(\frac{16}{3} \zeta_{2}+\right.\right.\right. \\
& \left.\left.\frac{80}{27}\right) \mathbf{N}_{-2}-\frac{188}{3}-8 \zeta_{2}\right) S_{1}+\left(\left(8 \zeta_{2}+\frac{184}{9}\right) \mathbf{N}_{+}+\frac{128}{9} \mathbf{N}_{+2}+\left(-\frac{184}{3}-8 \zeta_{2}\right) \mathbf{N}_{-}+32-\right. \\
& \left.\frac{16}{3} \mathbf{N}_{-2}\right) S_{2}+\left(-\frac{64}{3} \mathbf{N}_{-2}-\frac{58}{3} \mathbf{N}_{+}+\frac{16}{3} \mathbf{N}_{+2}+\frac{106}{3} \mathbf{N}_{-}\right) S_{3}+\left(-44 \mathbf{N}_{+}+44 \mathbf{N}_{-}\right) S_{4}+ \\
& \left(\frac{64}{3} \mathbf{N}_{+}-32-\frac{16}{3} \mathbf{N}_{-2}-\frac{16}{3} \mathbf{N}_{+2}+\frac{64}{3} \mathbf{N}_{-}\right) S_{1,-2}+\left(\frac{160}{3}-28 \mathbf{N}_{-}-\frac{8}{3} \mathbf{N}_{-2}-12 \mathbf{N}_{+}-\right. \\
& \left.\frac{32}{3} \mathbf{N}_{+\mathbf{2}}\right) S_{1,1}+\left(16-\frac{16}{3} \mathbf{N}_{+\mathbf{2}}-\frac{32}{3} \mathbf{N}_{-\mathbf{2}}-\frac{44}{3} \mathbf{N}_{+}+\frac{44}{3} \mathbf{N}_{-}\right) S_{2,1}+\left(24 \mathbf{N}_{-}-24 \mathbf{N}_{+}\right) S_{3,1}+ \\
& \left.\left(-\frac{16}{3} \mathbf{N}_{+2}+8-\frac{16}{3} \mathbf{N}_{-2}+\frac{4}{3} \mathbf{N}_{-}+\frac{4}{3} \mathbf{N}_{+}\right) S_{1,1,1}+\left(-8 \mathbf{N}_{+}+8 \mathbf{N}_{-}\right) S_{2,1,1}\right] \text {, } \\
& c_{T, \mathrm{~g}}^{(2)}(N)= \\
& \delta(N-2)\left[C_{F}^{2}\left\{\frac{1408}{15} \zeta_{3}+\frac{1120}{9} \zeta_{2}-\frac{140657}{405}\right\}+C_{F} C_{A}\left\{\frac{104}{9} \zeta_{2}+48 \zeta_{3}-\frac{26431}{81}\right\}\right]+ \\
& \theta(N-3)\left[C _ { F } ^ { 2 } \left\{\left(\left(36 \zeta_{2}+34-16 \zeta_{3}\right) \mathbf{N}_{+}-\frac{16}{5} \mathbf{N}_{+2}+\left(184+32 \zeta_{3}+208 \zeta_{2}\right) \mathbf{N}_{-}-\right.\right.\right. \\
& \left.\left(\frac{316}{5}+96 \zeta_{2}+64 \zeta_{3}\right) \mathbf{N}_{-2}-148 \zeta_{2}+48 \zeta_{3}-\frac{758}{5}\right) S_{1}+\left(\left(\frac{246}{5}+24 \zeta_{2}\right) \mathbf{N}_{+}+\frac{16}{5} \mathbf{N}_{+2}+\right. \\
& \left.\left(112 \zeta_{2}+\frac{274}{5}\right) \mathbf{N}_{-}+\left(\frac{144}{5}-64 \zeta_{2}\right) \mathbf{N}_{-2}-72 \zeta_{2}-136\right) S_{2}+\left(166 \mathbf{N}_{+}-230+\frac{16}{5} \mathbf{N}_{+2}-\right. \\
& \left.\frac{16}{5} \mathbf{N}_{+3}+64 \mathbf{N}_{-}\right) S_{3}+\left(-132+88 \mathbf{N}_{-}+44 \mathbf{N}_{+}\right) S_{4}+\left(96-96 \mathbf{N}_{-}+32 \mathbf{N}_{-2}-32 \mathbf{N}_{+}\right) S_{1,-3}+ \\
& \left(64 \mathbf{N}_{-}-\frac{16}{5} \mathbf{N}_{+3}-160-\frac{64}{5} \mathbf{N}_{-2}+\frac{64}{5} \mathbf{N}_{-3}+96 \mathbf{N}_{+}+\frac{16}{5} \mathbf{N}_{+2}\right) S_{1,-2}+\left(\left(16 \zeta_{2}+24\right) \mathbf{N}_{+}+\right. \\
& \left.\left(-8+64 \zeta_{2}\right) \mathbf{N}_{-}+\left(8-32 \zeta_{2}\right) \mathbf{N}_{-2}-24-48 \zeta_{2}\right) S_{1,1}+\left(-16+16 \mathbf{N}_{+}\right) S_{1,2}+\left(-96 \mathbf{N}_{-2}+\right. \\
& \left.64 \mathbf{N}_{+}-192+224 \mathbf{N}_{-}\right) S_{1,3}+\left(96 \mathbf{N}_{-2}-12 \mathbf{N}_{+}+92-176 \mathbf{N}_{-}\right) S_{2,1}+\left(-128 \mathbf{N}_{-}+\right. \\
& 128) S_{2,-2}+\left(64 \mathbf{N}_{-2}-128 \mathbf{N}_{-}+96-32 \mathbf{N}_{+}\right) S_{2,2}+\left(-8 \mathbf{N}_{+}-80 \mathbf{N}_{-}+64 \mathbf{N}_{-2}+24\right) S_{3,1}+ \\
& \left(-64 \mathbf{N}_{-2}+64 \mathbf{N}_{+}-192+192 \mathbf{N}_{-}\right) S_{1,1,-2}+\left(-28 \mathbf{N}_{+}-208 \mathbf{N}_{-}+96 \mathbf{N}_{-2}+140\right) S_{1,1,1}+ \\
& \left(-32 \mathbf{N}_{-}+16 \mathbf{N}_{-2}-8 \mathbf{N}_{+}+24\right) S_{1,1,2}+\left(72-24 \mathbf{N}_{+}-96 \mathbf{N}_{-}+48 \mathbf{N}_{-2}\right) S_{1,2,1}+ \\
& \left.\left(120+96 \mathbf{N}_{-2}-40 \mathbf{N}_{+}-176 \mathbf{N}_{-}\right) S_{2,1,1}+\left(80 \mathbf{N}_{-2}-40 \mathbf{N}_{+}+120-160 \mathbf{N}_{-}\right) S_{1,1,1,1}\right\}+ \\
& C_{A} C_{F}\left\{\left(\left(-\frac{1934}{27}+72 \zeta_{3}+\frac{8}{3} \zeta_{2}\right) \mathbf{N}_{+}+\left(-\frac{928}{27}-\frac{32}{3} \zeta_{2}\right) \mathbf{N}_{+2}+\left(\frac{440}{3} \zeta_{2}+240 \zeta_{3}+\right.\right.\right. \\
& \left.\left.\frac{5410}{27}\right) \mathbf{N}_{-}+\left(-\frac{248}{3} \zeta_{2}-96 \zeta_{3}-\frac{4438}{27}\right) \mathbf{N}_{-2}+70-216 \zeta_{3}-56 \zeta_{2}\right) S_{1}+\left(\left(-\frac{260}{9}-\right.\right.
\end{aligned}
$$




$$
\begin{aligned}
& \left.\left.48 \zeta_{2}\right) \mathbf{N}_{+}-\frac{256}{9} \mathbf{N}_{+\mathbf{2}}+\left(92-64 \zeta_{2}\right) \mathbf{N}_{-}+\left(\frac{496}{3}+64 \zeta_{2}\right) \mathbf{N}_{-2}-200+48 \zeta_{2}\right) S_{2}+ \\
& \left(\frac{464}{3} \mathbf{N}_{-2}-\frac{368}{3} \mathbf{N}_{-}+\frac{44}{3} \mathbf{N}_{+}-\frac{32}{3} \mathbf{N}_{+\mathbf{2}}-36\right) S_{3}+\left(248 \mathbf{N}_{+}-320 \mathbf{N}_{-2}+144 \mathbf{N}_{-}-72\right) S_{4}+ \\
& \left(48 \mathbf{N}_{+}-144+176 \mathbf{N}_{-}-80 \mathbf{N}_{-2}\right) S_{1,-3}+\left(\frac{32}{3} \mathbf{N}_{+2}-\frac{320}{3} \mathbf{N}_{-}+\frac{80}{3} \mathbf{N}_{-2}-\frac{200}{3} \mathbf{N}_{+}+\right. \\
& 136) S_{1,-2}+\left(\left(32 \zeta_{2}-\frac{28}{3}\right) \mathbf{N}_{+}+\frac{32}{3} \mathbf{N}_{+\mathbf{2}}+\left(128 \zeta_{2}-\frac{592}{3}\right) \mathbf{N}_{-}+\left(\frac{356}{3}-64 \zeta_{2}\right) \mathbf{N}_{-\mathbf{2}}+\right. \\
& \left.\frac{232}{3}-96 \zeta_{2}\right) S_{1,1}+\left(72-128 \mathbf{N}_{-}+64 \mathbf{N}_{-2}-8 \mathbf{N}_{+}\right) S_{1,2}+\left(96-32 \mathbf{N}_{+}-112 \mathbf{N}_{-}+\right. \\
& \left.48 \mathbf{N}_{-2}\right) S_{1,3}+\left(128 \mathbf{N}_{-}-64 \mathbf{N}_{-2}+32 \mathbf{N}_{+}-96\right) S_{2,-2}+\left(\frac{32}{3} \mathbf{N}_{+2}+\frac{496}{3} \mathbf{N}_{-2}+\frac{16}{3} \mathbf{N}_{+}-\right. \\
& \left.\frac{784}{3} \mathbf{N}_{-}+80\right) S_{2,1}+\left(96 \mathbf{N}_{-2}-48 \mathbf{N}_{+}+144-192 \mathbf{N}_{-}\right) S_{2,2}+\left(32-96 \mathbf{N}_{-2}-32 \mathbf{N}_{-}+\right. \\
& \left.96 \mathbf{N}_{+}\right) S_{3,1}+\left(64 \mathbf{N}_{-}-48-32 \mathbf{N}_{-2}+16 \mathbf{N}_{+}\right) S_{1,-2,1}+\left(-32 \mathbf{N}_{-}-16 \mathbf{N}_{+}+48\right) S_{1,1,-2}+ \\
& \left(\frac{344}{3} \mathbf{N}_{-\mathbf{2}}-\frac{80}{3} \mathbf{N}_{+}-\frac{632}{3} \mathbf{N}_{-}+112+\frac{32}{3} \mathbf{N}_{+\mathbf{2}}\right) S_{1,1,1}+\left(64 \mathbf{N}_{-\mathbf{2}}-128 \mathbf{N}_{-}+96-\right. \\
& \left.32 \mathbf{N}_{+}\right) S_{1,1,2}+\left(32 \mathbf{N}_{-2}-16 \mathbf{N}_{+}-64 \mathbf{N}_{-}+48\right) S_{1,2,1}+ \\
& \left.\left.\left(24 \mathbf{N}_{+}+24-32 \mathbf{N}_{-}-16 \mathbf{N}_{-2}\right) S_{2,1,1}+\left(24-32 \mathbf{N}_{-}+16 \mathbf{N}_{-2}-8 \mathbf{N}_{+}\right) S_{1,1,1,1}\right\}\right] \\
& c_{L, \mathrm{~ns}}^{(2)}(N)= \\
& \delta(N-2)\left[C_{F}^{2}\left\{\frac{48}{5} \zeta_{3}+\frac{33}{10}\right\}+C_{F} C_{A}\left\{-\frac{24}{5} \zeta_{3}+\frac{221}{10}\right\}-\frac{11}{3} C_{F} n_{f}\right]+ \\
& \theta(N-3)\left[C _ { F } ^ { 2 } \left\{\left(-10 \mathbf{N}_{+}+\frac{32}{5} \mathbf{N}_{+\mathbf{2}}+\left(-48 \zeta_{3}+39\right) \mathbf{N}_{-}-\frac{129}{5}-\frac{48}{5} \mathbf{N}_{-2}+48 \zeta_{3}\right) S_{1}+\right.\right. \\
& \left(\frac{8}{5} \mathbf{N}_{+}-\frac{32}{5} \mathbf{N}_{+\mathbf{2}}+\frac{82}{5} \mathbf{N}_{-}-\frac{48}{5} \mathbf{N}_{-\mathbf{2}}-2\right) S_{2}+\left(12 \mathbf{N}_{-}+32 \mathbf{N}_{+}-\frac{32}{5} \mathbf{N}_{+\mathbf{2}}+\frac{32}{5} \mathbf{N}_{+\mathbf{3}}-\right. \\
& 44) S_{3}+\left(16-16 \mathbf{N}_{-}\right) S_{1,-3}+\left(16 \mathbf{N}_{-}+\frac{48}{5} \mathbf{N}_{-2}+32 \mathbf{N}_{+}-\frac{32}{5} \mathbf{N}_{+2}+\frac{32}{5} \mathbf{N}_{+3}-\frac{48}{5} \mathbf{N}_{-\mathbf{3}}-\right. \\
& 48) S_{1,-2}+\left(14 \mathbf{N}_{-}-10-4 \mathbf{N}_{+}\right) S_{1,1}+\left(16-16 \mathbf{N}_{-}\right) S_{1,2}+\left(16 \mathbf{N}_{-}-16\right) S_{1,3}+ \\
& \left.\left(32-32 \mathbf{N}_{-}\right) S_{2,-2}+\left(4-4 \mathbf{N}_{-}\right) S_{2,1}+\left(-32+32 \mathbf{N}_{-}\right) S_{1,1,-2}+\left(-8 \mathbf{N}_{-}+8\right) S_{1,1,1}\right\}+ \\
& C_{F} C_{A}\left\{\left(-\frac{10}{3} \mathbf{N}_{+}-\frac{16}{5} \mathbf{N}_{+2}+\left(24 \zeta_{3}-\frac{389}{9}\right) \mathbf{N}_{-}+\frac{2023}{45}-24 \zeta_{3}+\frac{24}{5} \mathbf{N}_{-2}\right) S_{1}+\left(\frac{34}{3}+\right.\right. \\
& \left.\frac{24}{5} \mathbf{N}_{-2}-\frac{24}{5} \mathbf{N}_{+}+\frac{16}{5} \mathbf{N}_{+2}-\frac{218}{15} \mathbf{N}_{-}\right) S_{2}+\left(16+\frac{16}{5} \mathbf{N}_{+2}-\frac{16}{5} \mathbf{N}_{+3}-16 \mathbf{N}_{+}\right) S_{3}+
\end{aligned}
$$




$$
\begin{aligned}
& \left(8 \mathbf{N}_{-}-8\right) S_{1,-3}+\left(-\frac{24}{5} \mathbf{N}_{-2}-\frac{16}{5} \mathbf{N}_{+3}+\frac{16}{5} \mathbf{N}_{+2}+24-8 \mathbf{N}_{-}+\frac{24}{5} \mathbf{N}_{-\mathbf{3}}-16 \mathbf{N}_{+}\right) S_{1,-2}+ \\
& \left.\left(\frac{46}{3}-\frac{46}{3} \mathbf{N}_{-}\right) S_{1,1}+\left(-8 \mathbf{N}_{-}+8\right) S_{1,3}+\left(16 \mathbf{N}_{-}-16\right) S_{2,-2}+\left(16-16 \mathbf{N}_{-}\right) S_{1,1,-2}\right\}+ \\
& \left.C_{F} n_{f}\left\{\left(\frac{50}{9} \mathbf{N}_{-}-\frac{62}{9}+\frac{4}{3} \mathbf{N}_{+}\right) S_{1}+\left(\frac{4}{3} \mathbf{N}_{-}-\frac{4}{3}\right) S_{2}+\left(\frac{4}{3} \mathbf{N}_{-}-\frac{4}{3}\right) S_{1,1}\right\}\right] \\
& c_{L, \mathrm{ps}}^{(2)}(N)= \\
& -\frac{26}{3} \delta(N-2) C_{F} n_{f}+\theta(N-3) C_{F} n_{f}\left[\left(\frac{32}{3} \mathbf{N}_{-}-\frac{160}{3}+8 \mathbf{N}_{-2}+\frac{128}{3} \mathbf{N}_{+}-8 \mathbf{N}_{+2}\right) S_{1}+\right. \\
& \left(\frac{56}{3} \mathbf{N}_{+}-\frac{8}{3} \mathbf{N}_{+\mathbf{2}}-\frac{80}{3} \mathbf{N}_{-}+\frac{32}{3} \mathbf{N}_{-2}\right) S_{2}+\left(24-24 \mathbf{N}_{-}\right) S_{3}+ \\
& \left.\left(\frac{32}{3} \mathbf{N}_{+}-\frac{16}{3} \mathbf{N}_{-}-\frac{8}{3} \mathbf{N}_{+\mathbf{2}}-8+\frac{16}{3} \mathbf{N}_{-\mathbf{2}}\right) S_{1,1}+\left(8-8 \mathbf{N}_{-}\right) S_{2,1}\right] \text {, } \\
& c_{L, \mathrm{~g}}^{(2)}(N)= \\
& \delta(N-2)\left[C_{F}^{2}\left\{-\frac{96}{5} \zeta_{3}-\frac{108}{5}\right\}+\frac{272}{3} C_{F} C_{A}\right]+ \\
& \theta(N-3)\left[C _ { F } ^ { 2 } \left\{\left(\frac{56}{3} \mathbf{N}_{+}-\frac{176}{15}+\frac{96}{5} \mathbf{N}_{-2}-\frac{32}{15} \mathbf{N}_{+2}-24 \mathbf{N}_{-}\right) S_{1}+\left(\frac{64}{5} \mathbf{N}_{+}-\frac{40}{3}-\right.\right.\right. \\
& \left.\frac{104}{5} \mathbf{N}_{-}+\frac{96}{5} \mathbf{N}_{-\mathbf{2}}+\frac{32}{15} \mathbf{N}_{+\mathbf{2}}\right) S_{2}+\left(48-\frac{32}{15} \mathbf{N}_{+\mathbf{3}}-48 \mathbf{N}_{-}+\frac{32}{15} \mathbf{N}_{+\mathbf{2}}\right) S_{3}+ \\
& \left(\frac{32}{15} \mathbf{N}_{+\mathbf{2}}+\frac{32}{3} \mathbf{N}_{-}+\frac{64}{5} \mathbf{N}_{-\mathbf{2}}-\frac{64}{5} \mathbf{N}_{-\mathbf{3}}-\frac{32}{15} \mathbf{N}_{+\mathbf{3}}-\frac{32}{3}\right) S_{1,-2}+\left(16-16 \mathbf{N}_{-}\right) S_{2,1}+ \\
& \left.\left(8 \mathbf{N}_{+}-56 \mathbf{N}_{-}+32 \mathbf{N}_{-2}+16\right) S_{1,1}\right\}+ \\
& C_{F} C_{A}\left\{\left(-64 \mathbf{N}_{+}+\frac{32}{3} \mathbf{N}_{+\mathbf{2}}-\frac{160}{3}+256 \mathbf{N}_{-}-\frac{448}{3} \mathbf{N}_{-\mathbf{2}}\right) S_{1}+\left(\frac{16}{3} \mathbf{N}_{+\mathbf{2}}-\frac{112}{3} \mathbf{N}_{+}-80+\right.\right. \\
& \left.\frac{688}{3} \mathbf{N}_{-}-\frac{352}{3} \mathbf{N}_{-\mathbf{2}}\right) S_{2}+\left(-96+128 \mathbf{N}_{-2}-32 \mathbf{N}_{-}\right) S_{3}+\left(-64 \mathbf{N}_{-}+32 \mathbf{N}_{-2}+32\right) S_{1,-2}+ \\
& \left(-64+128 \mathbf{N}_{-}-64 \mathbf{N}_{-2}\right) S_{1,2}+\left(64 \mathbf{N}_{-}-64\right) S_{2,1}+\left(\frac{896}{3} \mathbf{N}_{-}-128+\frac{16}{3} \mathbf{N}_{+2}-\right. \\
& \left.\left.\left.\frac{464}{3} \mathbf{N}_{-2}-\frac{64}{3} \mathbf{N}_{+}\right) S_{1,1}+\left(-32 \mathbf{N}_{-2}+64 \mathbf{N}_{-}-32\right) S_{1,1,1}\right\}\right] \text {, } \\
& c_{A, \mathrm{~ns}}^{(2)}(N)= \\
& \delta(N-1) C_{F}\left[\frac{11}{3} C_{A}-\frac{2}{3} n_{f}\right](-12) \zeta_{3}+ \\
& \theta(N-2)\left[C _ { F } ^ { 2 } \left\{\left(\left(\frac{19}{2}-40 \zeta_{3}-12 \zeta_{2}\right) \mathbf{N}_{+}+\left(-16-40 \zeta_{3}-12 \zeta_{2}\right) \mathbf{N}_{-}-19+48 \zeta_{3}\right) S_{1}+\right.\right.
\end{aligned}
$$




$$
\begin{aligned}
& \left(\left(12 \zeta_{2}-92\right) \mathbf{N}_{+}+78+\left(4 \zeta_{2}-92\right) \mathbf{N}_{-}\right) S_{2}-\left(94 \mathbf{N}_{-}+66 \mathbf{N}_{+}\right) S_{4}+\left(20 \mathbf{N}_{-}-90 \mathbf{N}_{+}+\right. \\
& \left.16 \mathbf{N}_{+2}-12\right) S_{3}+\left(24+12 \mathbf{N}_{-}-12 \mathbf{N}_{+}\right) S_{1,2}+\left(-12 \mathbf{N}_{-}-12 \mathbf{N}_{+}-16\right) S_{1,3}+(16- \\
& \left.32 \mathbf{N}_{-}\right) S_{2,-2}+\left(-8 \mathbf{N}_{+}+44 \mathbf{N}_{-}-24\right) S_{2,1}+\left(36 \mathbf{N}_{-}+36 \mathbf{N}_{+}\right) S_{2,2}+\left(-32 \mathbf{N}_{-}+16-\right. \\
& \left.32 \mathbf{N}_{+}\right) S_{1,-3}+\left(16 \mathbf{N}_{-2}-24 \mathbf{N}_{+}+16 \mathbf{N}_{+2}-24 \mathbf{N}_{-}+16\right) S_{1,-2}+\left(\left(-32 \zeta_{2}-33\right) \mathbf{N}_{+}+\right. \\
& \left.28+\left(-22-32 \zeta_{2}\right) \mathbf{N}_{-}\right) S_{1,1}+\left(24 \mathbf{N}_{-}+16 \mathbf{N}_{+}\right) S_{2,1,1}-\left(16 \mathbf{N}_{-}+16 \mathbf{N}_{+}\right) S_{1,-2,1}+ \\
& \left(-32-16 \mathbf{N}_{-}-16 \mathbf{N}_{+}\right) S_{1,1,-2}+\left(28 \mathbf{N}_{-}+16 \mathbf{N}_{+}-8\right) S_{1,1,1}+\left(24 \mathbf{N}_{+}+24 \mathbf{N}_{-}\right) S_{1,1,2}+ \\
& \left(16 \mathbf{N}_{+}+16 \mathbf{N}_{-}\right) S_{1,2,1}+\left(24 \mathbf{N}_{+}+24 \mathbf{N}_{-}\right) S_{1,1,1,1}+72 S_{-4}-16 S_{-2}+16 S_{-3,1}+ \\
& \left.48 S_{-2,-2}-8 \mathbf{N}_{+} S_{3,1}+\frac{331}{8}-12 \zeta_{3}+59 \zeta_{2}\right\}+ \\
& C_{F} C_{A}\left\{\left(\left(-\frac{22}{3} \zeta_{2}+\frac{895}{54}-12 \zeta_{3}\right) \mathbf{N}_{+}+\left(-\frac{22}{3} \zeta_{2}+\frac{1415}{27}-12 \zeta_{3}\right) \mathbf{N}_{-}-\frac{95}{9}-24 \zeta_{3}\right) S_{1}+\right. \\
& \left(\left(\frac{193}{3}-8 \zeta_{2}\right) \mathbf{N}_{+}-\frac{250}{3}+\left(\frac{263}{3}-8 \zeta_{2}\right) \mathbf{N}_{-}\right) S_{2}+\left(-4-\frac{37}{3} \mathbf{N}_{-}+\frac{95}{3} \mathbf{N}_{+}-8 \mathbf{N}_{+\mathbf{2}}\right) S_{3}+ \\
& \left(8+4 \mathbf{N}_{-}+4 \mathbf{N}_{+}\right) S_{1,3}+\left(16 \mathbf{N}_{-}-8\right) S_{2,-2}+\left(-\frac{2}{3} \mathbf{N}_{-}+\frac{46}{3} \mathbf{N}_{+}\right) S_{2,1}+\left(4 \mathbf{N}_{+}+4 \mathbf{N}_{-}\right) S_{2,2}+ \\
& 36 \mathbf{N}_{-} S_{4}+\left(4 \mathbf{N}_{-}-4 \mathbf{N}_{+}\right) S_{3,1}-8 S_{-3,1}-24 S_{-2,-2}+\left(16 \mathbf{N}_{-}+16 \mathbf{N}_{+}-8\right) S_{1,-3}+\left(12 \mathbf{N}_{-}+\right. \\
& \left.12 \mathbf{N}_{+}-8 \mathbf{N}_{-\mathbf{2}}-8 \mathbf{N}_{+\mathbf{2}}-8\right) S_{1,-2}+\left(\left(8 \zeta_{2}+\frac{305}{9}\right) \mathbf{N}_{+}-36+\left(8 \zeta_{2}+\frac{386}{9}\right) \mathbf{N}_{-}\right) S_{1,1}+ \\
& \left(8 \mathbf{N}_{-}+8 \mathbf{N}_{+}\right) S_{1,-2,1}+\left(16+8 \mathbf{N}_{-}+8 \mathbf{N}_{+}\right) S_{1,1,-2}+\left(\frac{22}{3} \mathbf{N}_{+}+\frac{22}{3} \mathbf{N}_{-}\right) S_{1,1,1}-36 S_{-4}+ \\
& \left.8 S_{-2}+\left(-4 \mathbf{N}_{+}-4 \mathbf{N}_{-}\right) S_{1,1,2}+\left(4 \mathbf{N}_{+}+4 \mathbf{N}_{-}\right) S_{1,2,1}-\frac{5465}{72}+10 \zeta_{3}+7 \zeta_{2}\right\}+ \\
& C_{F} n_{f}\left\{\left(\left(\frac{4}{3} \zeta_{2}-\frac{131}{27}\right) \mathbf{N}_{+}+\frac{62}{9}+\left(\frac{4}{3} \zeta_{2}-\frac{302}{27}\right) \mathbf{N}_{-}\right) S_{1}+\left(\frac{16}{3}-\frac{22}{3} \mathbf{N}_{+}-\frac{26}{3} \mathbf{N}_{-}\right) S_{2}+\right. \\
& \left(-\frac{2}{3} \mathbf{N}_{+}-\frac{2}{3} \mathbf{N}_{-}\right) S_{3}+\left(-\frac{4}{3} \mathbf{N}_{+}-\frac{4}{3} \mathbf{N}_{-}\right) S_{2,1}+\left(-\frac{38}{9} \mathbf{N}_{+}+4-\frac{56}{9} \mathbf{N}_{-}\right) S_{1,1}+ \\
& \left.\left.\left(-\frac{4}{3} \mathbf{N}_{+}-\frac{4}{3} \mathbf{N}_{-}\right) S_{1,1,1}+\frac{457}{36}+8 \zeta_{3}-2 \zeta_{2}\right\}\right] \text {. }
\end{aligned}
$$

\section{Appendix C: The exact $x$-space results}

Here we write down the full $x$-space results of the time-like splitting functions and the coefficient functions $c_{T}, c_{L}$ and $c_{A}$ up to second order in $a_{\mathrm{s}}=\alpha_{\mathrm{s}} /(4 \pi)$, expressed in terms of harmonic poly- 
logarithms in the notation $H_{m_{1}, \ldots, m_{w}}(x), m_{j}=0, \pm 1$ of Ref. [67]. Below we use the short-hand notation

$$
H_{\underbrace{0, \ldots, 0}_{m}} \pm 1, \underbrace{0, \ldots, 0,}_{n} \pm 1, \ldots(x)=H_{ \pm(m+1), \pm(n+1), \ldots}(x),
$$

suppress the argument $x$ for brevity and define

$$
\begin{aligned}
& p_{\mathrm{qq}}(x)=2(1-x)^{-1}-1-x, \\
& p_{\mathrm{gg}}(x)=(1-x)^{-1}+x^{-1}-2+x-x^{2} .
\end{aligned}
$$

All divergences for $x \rightarrow 1$ are understood in the sense of + -distributions.

The well known results at $\mathrm{LO}$ are:

$$
\begin{aligned}
& P_{\mathrm{qq}}^{(0)}(x)=C_{F}\left(2 p_{\mathrm{qq}}(x)+3 \delta(1-x)\right), \\
& P_{\mathrm{qg}}^{(0)}(x)=n_{f}\left(2-4 x+4 x^{2}\right), \\
& P_{\mathrm{gq}}^{(0)}(x)=C_{F}\left(-4+\frac{4}{x}+2 x\right), \\
& P_{\mathrm{gg}}^{(0)}(x)=C_{A}\left(4 p_{\mathrm{gg}}(x)+\frac{11}{3} \delta(1-x)\right)-n_{f} \frac{2}{3} \delta(1-x) .
\end{aligned}
$$

The NLO splitting functions read ${ }^{4}$ :

$$
\begin{aligned}
& P_{\mathrm{ns}}^{(1)+}(x)= \\
& C_{F}{ }^{2}\left\{p_{\mathrm{qq}}(x)\left(-8 \mathrm{H}_{2}+6 \mathrm{H}_{0}-16 \mathrm{H}_{0,0}-8 \mathrm{H}_{1,0}\right)+p_{\mathrm{qq}}(-x)\left(-8 \zeta_{2}+8 \mathrm{H}_{0,0}-16 \mathrm{H}_{-1,0}\right)+\right. \\
& \left.(-6+2 x) \mathrm{H}_{0}+(4+4 x) \mathrm{H}_{0,0}-4+4 x+\left(-12 \zeta_{2}+\frac{3}{2}+24 \zeta_{3}\right) \delta(1-x)\right\}+ \\
& C_{A} C_{F}\left\{p_{\mathrm{qq}}(x)\left(4 \mathrm{H}_{0,0}-4 \zeta_{2}+\frac{134}{9}+\frac{22}{3} \mathrm{H}_{0}\right)+p_{\mathrm{qq}}(-x)\left(-4 \mathrm{H}_{0,0}+8 \mathrm{H}_{-1,0}+4 \zeta_{2}\right)+\right. \\
& \left.\quad \frac{56}{3}(1-x)+\left(\frac{44}{3} \zeta_{2}+\frac{17}{6}-12 \zeta_{3}\right) \delta(1-x)\right\}+ \\
& n_{f} C_{F}\left\{p_{\mathrm{qq}}(x)\left(-\frac{4}{3} \mathrm{H}_{0}-\frac{20}{9}\right)-\frac{8}{3}(1-x)+\left(-\frac{1}{3}-\frac{8}{3} \zeta_{2}\right) \delta(1-x)\right\} \\
& P_{\mathrm{ns}}^{(1)-}(x)= \\
& \quad C_{F}{ }^{2}\left\{p_{\mathrm{qq}}(x)\left(6 \mathrm{H}_{0}-8 \mathrm{H}_{2}-8 \mathrm{H}_{1,0}-16 \mathrm{H}_{0,0}\right)+p_{\mathrm{qq}}(-x)\left(16 \mathrm{H}_{-1,0}-8 \mathrm{H}_{0,0}+8 \zeta_{2}\right)+\right. \\
& \left.\quad(-14 x-22) \mathrm{H}_{0}+(4+4 x) \mathrm{H}_{0,0}+36 x-36+\left(\frac{3}{2}-12 \zeta_{2}+24 \zeta_{3}\right) \delta(1-x)\right\}+
\end{aligned}
$$

\footnotetext{
${ }^{4}$ We quote $P_{\mathrm{qg}}^{(1)}$ and $P_{\mathrm{gg}}^{(1)}$ here for completeness (see discussion in Section 4 .
} 


$$
\begin{aligned}
& C_{F} C_{A}\left\{p_{\mathrm{qq}}(x)\left(-4 \zeta_{2}+4 \mathrm{H}_{0,0}+\frac{22}{3} \mathrm{H}_{0}+\frac{134}{9}\right)+\left(-4 \zeta_{2}-8 \mathrm{H}_{-1,0}+4 \mathrm{H}_{0,0}\right) p_{\mathrm{qq}}(-x)+\right. \\
& \left.(8 x+8) \mathrm{H}_{0}-\frac{104}{3} x+\frac{104}{3}+\left(-12 \zeta_{3}+\frac{17}{6}+\frac{44}{3} \zeta_{2}\right) \delta(1-x)\right\}+ \\
& C_{F} n_{f}\left\{p_{\mathrm{qq}}(x)\left(-\frac{4}{3} \mathrm{H}_{0}-\frac{20}{9}\right)+\frac{8}{3} x-\frac{8}{3}-\left(\frac{1}{3}+\frac{8}{3} \zeta_{2}\right) \delta(1-x)\right\} \text {, } \\
& P_{\mathrm{qq}}^{(1) \mathrm{s}}(x)=n_{f} C_{F}\left\{8(1+x) \mathrm{H}_{0,0}-\left(\frac{32}{3} x^{2}+20+36 x\right) \mathrm{H}_{0}+\frac{16(x-1)\left(14 x^{2}+23 x+5\right)}{9 x}\right\} \\
& P_{\mathrm{qg}}^{(1)}(x)= \\
& n_{f} C_{F}\left\{\left(8 x-10+8 x^{2}\right) \mathrm{H}_{0}+\left(8 x^{2}+12-8 x\right) \mathrm{H}_{1}+\left(8 x-4-16 x^{2}\right) \mathrm{H}_{0,0}+\right. \\
& \left.\left(-16 x+16 x^{2}+8\right)\left(\mathrm{H}_{2}-3 \mathrm{H}_{1,0}-\mathrm{H}_{1,1}-\zeta_{2}\right)-40 x^{2}-24+46 x\right\}+ \\
& n_{f} C_{A}\left\{\left(-\frac{136}{3} x-\frac{16}{3} x^{2}-\frac{16}{3}\right) \mathrm{H}_{0}+\left(\frac{40}{3} x-\frac{40}{3} x^{2}-\frac{44}{3}\right) \mathrm{H}_{1}+\right. \\
& \left(-16 x+16 x^{2}+8\right)\left(\mathrm{H}_{1,1}-2 \mathrm{H}_{2}+2 \mathrm{H}_{1,0}\right)+\left(-16 x-8-16 x^{2}\right) \mathrm{H}_{-1,0}+ \\
& \left.(8+48 x) \mathrm{H}_{0,0}-16 \zeta_{2} x+\frac{4\left(569 x^{2}-20+178 x^{3}+13 x\right)}{9 x}\right\}+ \\
& n_{f}^{2}\left\{\left(\frac{16}{3} x^{2}+\frac{8}{3}-\frac{16}{3} x\right)\left(\mathrm{H}_{1}-\mathrm{H}_{0}\right)-\frac{40}{9}+\frac{32}{9} x-\frac{32}{9} x^{2}\right\} \text {, } \\
& P_{\mathrm{gq}}^{(1)}(x)= \\
& C_{F}^{2}\left\{(-32+2 x) \mathrm{H}_{0}-8 x \mathrm{H}_{1}+\frac{8\left(-2 x+x^{2}+2\right)}{x}\left(\mathrm{H}_{1,1}+2 \mathrm{H}_{1,0}-2 \mathrm{H}_{2}\right)+\right. \\
& \left.(8-4 x) \mathrm{H}_{0,0}+18 x-2\right\}+ \\
& C_{A} C_{F}\left\{\frac{4\left(27 x^{2}+8 x^{3}+24 x-18\right)}{3 x} \mathrm{H}_{0}+8 x \mathrm{H}_{1}+\frac{8\left(-2 x+x^{2}+2\right)}{x}\left(\mathrm{H}_{2}-3 \mathrm{H}_{1,0}-\mathrm{H}_{1,1}\right)+\right. \\
& \left.\frac{8\left(2 x+x^{2}+2\right)}{x} \mathrm{H}_{-1,0}-\frac{8\left(2 x+3 x^{2}+4\right)}{x} \mathrm{H}_{0,0}+16 \zeta_{2}-\frac{4\left(-45 x+9 x^{2}-17+44 x^{3}\right)}{9 x}\right\} \text {, } \\
& P_{\mathrm{gg}}^{(1)}(x)= \\
& n_{f} C_{F}\left\{\frac{4\left(21 x^{2}+8 x^{3}+15 x+8\right)}{3 x} \mathrm{H}_{0}+(8 x+8) \mathrm{H}_{0,0}-\right. \\
& \left.\frac{8(x-1)\left(41 x^{2}+14 x+23\right)}{9 x}-2 \delta(1-x)\right\}+ \\
& C_{A}^{2}\left\{p_{\mathrm{gg}}(x)\left(\frac{268}{9}-16 \mathrm{H}_{2}-8 \zeta_{2}+\frac{88}{3} \mathrm{H}_{0}-24 \mathrm{H}_{0,0}-16 \mathrm{H}_{1,0}\right)+\right.
\end{aligned}
$$




$$
\begin{aligned}
& \left(-8 \zeta_{2}+8 \mathrm{H}_{0,0}-16 \mathrm{H}_{-1,0}\right) p_{\mathrm{gg}}(-x)-\frac{4\left(44-11 x+25 x^{2}\right)}{3 x} \mathrm{H}_{0}+(-32-32 x) \mathrm{H}_{0,0}+ \\
& \left.\frac{2(x-1)\left(134 x^{2}-109 x+134\right)}{9 x}+\left(12 \zeta_{3}+\frac{32}{3}\right) \delta(1-x)\right\}+ \\
& n_{f} C_{A}\left\{\left(-\frac{16}{3} \mathrm{H}_{0}-\frac{40}{9}\right) p_{\mathrm{gg}}(x)-\left(\frac{8}{3}+\frac{8}{3} x\right) \mathrm{H}_{0}+\frac{4(x-1)\left(13 x^{2}+4 x+13\right)}{9 x}-\frac{8}{3} \delta(1-x)\right\} .
\end{aligned}
$$

The coefficient functions at order $O\left(\alpha_{\mathrm{s}}\right)$ read:

$$
\begin{aligned}
& c_{T, \mathrm{q}}^{(1)}(x)=C_{F}\left(p_{\mathrm{qq}}(x)\left(-\frac{3}{2}+4 \mathrm{H}_{0}-2 \mathrm{H}_{1}\right)-\frac{9}{2} x+\frac{3}{2}+\left(8 \zeta_{2}-9\right) \delta(1-x)\right), \\
& c_{L, \mathrm{q}}^{(1)}(x)=2 C_{F} \\
& c_{A, \mathrm{q}}^{(1)}(x)-c_{T, \mathrm{q}}^{(1)}(x)=C_{F}(2 x-2) \\
& c_{T, \mathrm{~g}}^{(1)}(x)=C_{F}\left(\frac{8\left(-2 x+2+x^{2}\right)}{x} \mathrm{H}_{0}-\frac{4\left(-2 x+2+x^{2}\right)}{x} \mathrm{H}_{1}+\frac{8(-1+x)}{x}\right) \\
& c_{L, \mathrm{~g}}^{(1)}(x)=C_{F} \frac{8(1-x)}{x} .
\end{aligned}
$$

The coefficient functions at order $O\left(\alpha_{\mathrm{s}}^{2}\right)$ read:

$$
\begin{aligned}
c_{T, \mathrm{~ns}}^{(2)}(x)= & (\mathrm{C} .18) \\
& C_{F}^{2}\left\{p _ { \mathrm { qq } } ( x ) \left(\left(20 \zeta_{2}-53\right) \mathrm{H}_{0}+6 \zeta_{2}+6 \mathrm{H}_{2}+\frac{27}{2} \mathrm{H}_{1}+4 \mathrm{H}_{3}+12 \mathrm{H}_{1,0}+\frac{51}{4}-18 \mathrm{H}_{1,1}+\right.\right. \\
& 33 \mathrm{H}_{0,0}-76 \zeta_{3}+20 \mathrm{H}_{1,0,0}+24 \mathrm{H}_{1,1,0}-24 \mathrm{H}_{1,1,1}+16 \mathrm{H}_{1,2}-36 \mathrm{H}_{2,0}+24 \mathrm{H}_{-2,0}+20 \mathrm{H}_{2,1}- \\
& \left.80 \mathrm{H}_{0,0,0}\right)+p_{\mathrm{qq}}(-x)\left(-32 \mathrm{H}_{-1} \zeta_{2}+\left(-8 \zeta_{2}-8\right) \mathrm{H}_{0}-8 \mathrm{H}_{-2,0}-8 \mathrm{H}_{3}+36 \mathrm{H}_{0,0,0}-\right. \\
& \left.32 \mathrm{H}_{-1,-1,0}-24 \mathrm{H}_{-1,0,0}+16 \mathrm{H}_{-1,2}+28 \zeta_{3}\right)+(-8 x-8) \zeta_{2} \mathrm{H}_{-1}+\left((8 x+8) \zeta_{2}+\right. \\
& -59 x^{2}+48+48 x^{3}+71 x \\
5 x & \mathrm{H}_{0}+\left((-8+8 x) \zeta_{2}-\frac{75}{2} x+\frac{53}{2}\right) \mathrm{H}_{1}+(18+2 x) \mathrm{H}_{2}- \\
& (12+12 x) \mathrm{H}_{3}-\frac{8(x+1)\left(6 x^{4}-6 x^{3}+x^{2}-6 x+6\right)}{5 x^{2}} \mathrm{H}_{-1,0}+\left(-33+3 x+\frac{48}{5} x^{3}\right) \mathrm{H}_{0,0}+ \\
& (40 x-16) \mathrm{H}_{1,0}+16 \mathrm{H}_{-2,0}+(-10 x-2) \mathrm{H}_{1,1}+(4 x+4) \mathrm{H}_{2,1}+(-16-16 x) \mathrm{H}_{-1,-1,0}+ \\
& (8 x+8) \mathrm{H}_{-1,0,0}+(22 x+22) \mathrm{H}_{0,0,0}+(-8 x+8) \mathrm{H}_{1,0,0}+\left(6 x-\frac{48}{5} x^{3}-18\right) \zeta_{2}+ \\
& \left.(8+24 x) \zeta_{3}+\frac{3\left(271 x-101 x^{2}-64+64 x^{3}\right)}{20 x}+\left(\frac{331}{8}+30 \zeta_{2}^{2}-78 \zeta_{3}-39 \zeta_{2}\right) \delta(1-x)\right\}+ \\
C_{F} C_{A}\left\{p _ { \mathrm { qq } } ( x ) \left(\left(\frac{103}{3}-16 \zeta_{2}\right) \mathrm{H}_{0}-\left(4 \zeta_{2}+\frac{367}{18}\right) \mathrm{H}_{1}-4 \mathrm{H}_{2,0}-\frac{3155}{108}-\frac{22}{3} \mathrm{H}_{1,1}+14 \zeta_{3}-\right.\right. &
\end{aligned}
$$




$$
\begin{aligned}
& \left.12 \mathrm{H}_{-2,0}-\frac{11}{3} \mathrm{H}_{0,0}-8 \mathrm{H}_{1,0,0}-4 \mathrm{H}_{1,1,0}+\frac{22}{3} \mathrm{H}_{2}+18 \mathrm{H}_{0,0,0}+4 \mathrm{H}_{1,2}\right)+p_{\mathrm{qq}}(-x)\left(16 \mathrm{H}_{-1} \zeta_{2}+\right. \\
& \left.\left(4 \zeta_{2}+4\right) \mathrm{H}_{0}-18 \mathrm{H}_{0,0,0}+4 \mathrm{H}_{-2,0}-14 \zeta_{3}+12 \mathrm{H}_{-1,0,0}-8 \mathrm{H}_{-1,2}+16 \mathrm{H}_{-1,-1,0}+4 \mathrm{H}_{3}\right)+ \\
& (4 x+4) \zeta_{2} \mathrm{H}_{-1}-\frac{2\left(-13 x^{2}+36-108 x+36 x^{3}\right)}{15 x} \mathrm{H}_{0}+\left((4-4 x) \zeta_{2}-\frac{139}{6}+\frac{85}{6} x\right) \mathrm{H}_{1}- \\
& 8 \mathrm{H}_{-2,0}+\frac{4(x+1)\left(6 x^{4}-6 x^{3}+x^{2}-6 x+6\right)}{5 x^{2}} \mathrm{H}_{-1,0}-\frac{4}{5} x\left(-5+6 x^{2}\right) \mathrm{H}_{0,0}+ \\
& (8 x+8) \mathrm{H}_{-1,-1,0}+(-4-4 x) \mathrm{H}_{-1,0,0}+(4 x-4) \mathrm{H}_{1,0,0}+\frac{4}{5} x\left(-5+6 x^{2}\right) \zeta_{2}-8 \zeta_{3} x- \\
& \left.\frac{\left(2039 x^{2}-864+864 x^{3}+4411 x\right)}{180 x}+\left(-\frac{49}{5} \zeta_{2}^{2}-\frac{5465}{72}+\frac{140}{3} \zeta_{3}+\frac{215}{3} \zeta_{2}\right) \delta(1-x)\right\}+ \\
& C_{F} n_{f}\left\{p_{\mathrm{qq}}(x)\left(-\frac{16}{3} \mathrm{H}_{0}+\frac{4}{3} \mathrm{H}_{1,1}+\frac{29}{9} \mathrm{H}_{1}+\frac{247}{54}-\frac{4}{3} \mathrm{H}_{2}+\frac{2}{3} \mathrm{H}_{0,0}\right)+\left(-2+\frac{2}{3} x\right) \mathrm{H}_{0}+\right. \\
& \left.\left(\frac{5}{3}+\frac{1}{3} x\right) \mathrm{H}_{1}+\frac{43}{18}+\frac{71}{18} x+\left(\frac{4}{3} \zeta_{3}+\frac{457}{36}-\frac{38}{3} \zeta_{2}\right) \delta(1-x)\right\} \\
& c_{T, \mathrm{ps}}^{(2)}(x)= \\
& C_{F} n_{f}\left\{\left((16+16 x) \zeta_{2}-\frac{8\left(16 x^{3}+75 x+6+39 x^{2}\right)}{9 x}\right) \mathrm{H}_{0}-(24 x+24) \mathrm{H}_{3}-\right. \\
& \frac{4(x-1)\left(8 x^{2}+25 x+2\right)}{3 x} \mathrm{H}_{1}+\frac{4(x+1)\left(4 x^{2}+11 x-8\right)}{3 x} \mathrm{H}_{2}-\frac{16(x+1)^{3}}{3 x} \mathrm{H}_{-1,0}+ \\
& \frac{2\left(32-21 x^{2}+8 x^{3}-21 x\right)}{3 x} \mathrm{H}_{0,0}-\frac{4(x-1)\left(4 x^{2}+7 x+4\right)}{3 x} \mathrm{H}_{1,1}+(8+8 x) \mathrm{H}_{2,1}+ \\
& \left.(44 x+44) \mathrm{H}_{0,0,0}+(-8-32 x) \zeta_{2}+(16+16 x) \zeta_{3}+\frac{2(x-1)\left(256 x^{2}+571 x+40\right)}{27 x}\right\}, \\
& c_{T, \mathrm{~g}}^{(2)}(x)= \\
& C_{F}^{2}\left\{\frac{32(x+1)^{2}}{x} \zeta_{2} \mathrm{H}_{-1}+\left((-16 x+32) \zeta_{2}-\frac{2\left(-209 x-72+131 x^{2}+8 x^{3}\right)}{5 x}\right) \mathrm{H}_{0}+\right. \\
& \left(-\frac{8\left(5 x^{2}-10 x+6\right)}{x} \zeta_{2}+\frac{8\left(3 x^{2}-1\right)}{x}\right) \mathrm{H}_{1}+\frac{4\left(3 x^{2}-20 x+24\right)}{x} \mathrm{H}_{2}-\frac{8\left(-2 x+x^{2}+8\right)}{x} \mathrm{H}_{3}- \\
& 128 \mathrm{H}_{-2,0}+\frac{16\left(-20 x^{2}-30 x^{3}+x^{5}-4\right)}{5 x^{2}} \mathrm{H}_{-1,0}+\left(-64+166 x-\frac{16}{5} x^{3}\right) \mathrm{H}_{0,0}-16 \mathrm{H}_{1,0} x- \\
& \frac{4\left(-28 x+7 x^{2}+24\right)}{x} \mathrm{H}_{1,1}+\frac{24\left(-2 x+2+x^{2}\right)}{x} \mathrm{H}_{1,2}-\frac{32\left(-2 x+2+x^{2}\right)}{x} \mathrm{H}_{2,0}+ \\
& \frac{8\left(-10 x+12+5 x^{2}\right)}{x} \mathrm{H}_{2,1}+\frac{64(x+1)^{2}}{x} \mathrm{H}_{-1,-1,0}-\frac{32(x+1)^{2}}{x} \mathrm{H}_{-1,0,0}+(-44 x+88) \mathrm{H}_{0,0,0}+ \\
& \frac{32\left(2 x^{2}-4 x+3\right)}{x} \mathrm{H}_{1,0,0}+\frac{8\left(-2 x+2+x^{2}\right)}{x} \mathrm{H}_{1,1,0}-\frac{40\left(-2 x+2+x^{2}\right)}{x} \mathrm{H}_{1,1,1}+
\end{aligned}
$$




$$
\begin{aligned}
& \left.\left(-32+\frac{16}{5} x^{3}-72 x\right) \zeta_{2}-\frac{16\left(4+5 x^{2}-2 x\right)}{x} \zeta_{3}-\frac{2\left(302 x+8 x^{3}-77 x^{2}-158\right)}{5 x}\right\}+ \\
& C_{F} C_{A}\left\{\left(-\frac{16\left(8 x+x^{2}-2\right)}{x} \zeta_{2}+\frac{4\left(579 x+372+64 x^{3}+129 x^{2}\right)}{9 x}\right) \mathrm{H}_{0}+\right. \\
& \frac{8\left(4+x^{2}+2 x\right)}{x} \zeta_{2} \mathrm{H}_{-1}+\left(\frac{8\left(3 x^{2}-6 x+4\right)}{x} \zeta_{2}+\frac{4\left(8 x^{3}+x^{2}-89+59 x\right)}{3 x}\right) \mathrm{H}_{1}- \\
& \frac{16\left(3 x^{2}+18 x-31+2 x^{3}\right)}{3 x} \mathrm{H}_{2}+\frac{32\left(4 x+3+3 x^{2}\right)}{x} \mathrm{H}_{3}+\frac{32\left(2 x+2+x^{2}\right)}{x} \mathrm{H}_{-2,0}+ \\
& \frac{8\left(4 x^{3}+10+30 x+21 x^{2}\right)}{3 x} \mathrm{H}_{-1,0}-\frac{16\left(2 x+2+x^{2}\right)}{x} \mathrm{H}_{-1,2}-\frac{4\left(24 x-3 x^{2}+116+8 x^{3}\right)}{3 x} \mathrm{H}_{0,0}+ \\
& \frac{8\left(8+x^{2}-8 x\right)}{x} \mathrm{H}_{1,0}+\frac{8\left(36 x-6 x^{2}+4 x^{3}-43\right)}{3 x} \mathrm{H}_{1,1}+\frac{16\left(-2 x+2+x^{2}\right)}{x} \mathrm{H}_{1,2}- \\
& \frac{48\left(-2 x+2+x^{2}\right)}{x} \mathrm{H}_{2,0}-\frac{8\left(3 x^{2}+6 x+2\right)}{x} \mathrm{H}_{2,1}+(-16 x-32) \mathrm{H}_{-1,-1,0}+ \\
& \frac{16\left(5+6 x+3 x^{2}\right)}{x} \mathrm{H}_{-1,0,0}-\frac{8\left(40+22 x+31 x^{2}\right)}{x} \mathrm{H}_{0,0,0}-\frac{16\left(2 x^{2}-4 x+3\right)}{x} \mathrm{H}_{1,0,0}+ \\
& \frac{32\left(-2 x+2+x^{2}\right)}{x} \mathrm{H}_{1,1,0}-\frac{8\left(-2 x+2+x^{2}\right)}{x} \mathrm{H}_{1,1,1}+\frac{8\left(-7+8 x^{2}+4 x\right)}{x} \zeta_{2}- \\
& \left.\frac{8\left(17 x^{2}+30-8 x\right)}{x} \zeta_{3}-\frac{2\left(486 x-2219+464 x^{3}+1431 x^{2}\right)}{27 x}\right\}
\end{aligned}
$$

$c_{L, \mathrm{nS}}^{(2)}(x)=$

$$
\begin{aligned}
& C_{F}^{2}\left\{16 \mathrm{H}_{-1} \zeta_{2}+\frac{2\left(17 x-24+12 x^{2}+16 x^{3}\right)}{5 x} \mathrm{H}_{0}+\left(16 \zeta_{2}-4 x-14\right) \mathrm{H}_{1}-4 \mathrm{H}_{2}-\right. \\
& \frac{16\left(2 x^{5}+5 x^{2}-3+10 x^{3}\right)}{5 x^{2}} \mathrm{H}_{-1,0}+\left(\frac{32}{5} x^{3}-12+32 x\right) \mathrm{H}_{0,0}-16 \mathrm{H}_{1,0}+8 \mathrm{H}_{1,1}-32 \mathrm{H}_{-2,0}+ \\
& \left.32 \mathrm{H}_{-1,-1,0}-16 \mathrm{H}_{-1,0,0}-16 \mathrm{H}_{1,0,0}+\left(4-\frac{32}{5} x^{3}-32 x\right) \zeta_{2}+\frac{48-147 x-18 x^{2}+32 x^{3}}{5 x}\right\}+ \\
& C_{F} C_{A}\left\{-8 \mathrm{H}_{-1} \zeta_{2}-\frac{2\left(-12 x^{2}-36+73 x+24 x^{3}\right)}{15 x} \mathrm{H}_{0}+\left(\frac{46}{3}-8 \zeta_{2}\right) \mathrm{H}_{1}+\right. \\
& \frac{8\left(2 x^{5}+5 x^{2}-3+10 x^{3}\right)}{5 x^{2}} \mathrm{H}_{-1,0}-\frac{16}{5} x\left(x^{2}+5\right) \mathrm{H}_{0,0}+16 \mathrm{H}_{-2,0}-16 \mathrm{H}_{-1,-1,0}+8 \mathrm{H}_{-1,0,0}+ \\
& \left.8 \mathrm{H}_{1,0,0}+\frac{16}{5} x\left(x^{2}+5\right) \zeta_{2}-\frac{144 x^{3}+294 x^{2}+216-1729 x}{45 x}\right\}+ \\
& C_{F} n_{f}\left\{-\frac{4}{3} \mathrm{H}_{1}-\frac{50}{9}+\frac{4}{3} \mathrm{H}_{0}+\frac{4}{3} x\right\}
\end{aligned}
$$

$c_{L, \mathrm{ps}}^{(2)}(x)=$

$$
C_{F} n_{f}\left\{\frac{8\left(-6 x^{2}+x^{3}-6 x+4\right)}{3 x} \mathrm{H}_{0}-\frac{8(x-1)\left(x^{2}-2 x-2\right)}{3 x} \mathrm{H}_{1}-8 \mathrm{H}_{2}+\right.
$$




$$
\left.24 \mathrm{H}_{0,0}+8 \zeta_{2}-\frac{8(x-1)\left(3 x^{2}-10 x-3\right)}{3 x}\right\},
$$

$$
\begin{aligned}
& c_{L, \mathrm{~g}}^{(2)}(x)= \\
& C_{F}{ }^{2}\left\{\frac{8(x+4)(x-1)}{x} \mathrm{H}_{1}-\frac{8\left(28 x^{2}-36+3 x+4 x^{3}\right)}{15 x} \mathrm{H}_{0}-\left(\frac{32}{15} x^{3}-48\right) \mathrm{H}_{0,0}-16 \mathrm{H}_{2}+\right. \\
& \left.\frac{32(x+1)\left(x^{4}-x^{3}+x^{2}-6 x+6\right)}{15 x^{2}} \mathrm{H}_{-1,0}+\left(16+\frac{32}{15} x^{3}\right) \zeta_{2}-\frac{8(x-1)\left(4 x^{2}-27 x-36\right)}{15 x}\right\}+ \\
& C_{F} C_{A}\left\{-\frac{16\left(22-6 x^{2}-21 x+x^{3}\right)}{3 x} \mathrm{H}_{0}+\frac{16(x-1)\left(x^{2}-2 x-29\right)}{3 x} \mathrm{H}_{1}+64 \mathrm{H}_{2}+\right. \\
& \frac{32(x+1)}{x} \mathrm{H}_{-1,0}-\frac{32(3 x+4)}{x} \mathrm{H}_{0,0}+\frac{64(x-1)}{x} \mathrm{H}_{1,0}-\frac{32(x-1)}{x} \mathrm{H}_{1,1}- \\
& \left.\frac{32(2 x-1)}{x} \zeta_{2}+\frac{32(x-1)\left(x^{2}-4 x-14\right)}{3 x}\right\},
\end{aligned}
$$

$$
\begin{aligned}
& c_{A, \mathrm{q}}^{(2)}(x)-c_{T, \mathrm{q}}^{(2)}(x)={ }_{F}{ }^{2}\left\{p _ { \mathrm { qq } } ( - x ) \left(\left(16 \zeta_{2}+16\right) \mathrm{H}_{0}+48 \mathrm{H}_{-1,0,0}-32 \mathrm{H}_{-1,2}+16 \mathrm{H}_{3}-\right.\right. \\
&\left.72 \mathrm{H}_{0,0,0}+16 \mathrm{H}_{-2,0}+64 \mathrm{H}_{-1,-1,0}-56 \zeta_{3}+64 \mathrm{H}_{-1} \zeta_{2}\right)-\frac{2\left(153 x+24-107 x^{2}+24 x^{3}\right)}{5 x} \mathrm{H}_{0}+ \\
&\left((16-16 x) \zeta_{2}+18 x-18\right) \mathrm{H}_{1}+(20+12 x) \mathrm{H}_{2}+(16-16 x) \mathrm{H}_{1,0}+(-8+8 x) \mathrm{H}_{1,1}+ \\
&(-16+16 x) \mathrm{H}_{-2,0}+\frac{16(x+1)\left(3 x^{2}+x+3\right)\left(x^{2}-3 x+1\right)}{5 x^{2}} \mathrm{H}_{-1,0}+ \\
&\left(-20-44 x+16 x^{2}-\frac{48}{5} x^{3}\right) \mathrm{H}_{0,0}-(16-16 x) \mathrm{H}_{1,0,0}+\left(-16 x^{2}-20 x-28+\frac{48}{5} x^{3}\right) \zeta_{2}+ \\
&\left.(16-16 x) \zeta_{3}-\frac{(x-1)\left(48 x^{2}-139 x+48\right)}{5 x}\right\}+ \\
& C_{F} C_{A}\left\{p _ { \mathrm { qq } } ( - x ) \left(\left(-8 \zeta_{2}-8\right) \mathrm{H}_{0}+28 \zeta_{3}+36 \mathrm{H}_{0,0,0}-8 \mathrm{H}_{3}-32 \mathrm{H}_{-1,-1,0}+16 \mathrm{H}_{-1,2}-\right.\right. \\
&\left.24 \mathrm{H}_{-1,0,0}-8 \mathrm{H}_{-2,0}-32 \mathrm{H}_{-1} \zeta_{2}\right)+\frac{4\left(18-104 x^{2}+18 x^{3}+161 x\right)}{15 x} \mathrm{H}_{0}+\left((-8+8 x) \zeta_{2}-\right. \\
&\left.\frac{2}{3} x+\frac{2}{3}\right) \mathrm{H}_{1}+(-8 x-8) \mathrm{H}_{2}+(8-8 x) \mathrm{H}_{-2,0}-\frac{8(x+1)\left(3 x^{2}+x+3\right)\left(x^{2}-3 x+1\right)}{5 x^{2}} \mathrm{H}_{-1,0}+ \\
& C_{F} n_{f}\left\{\left(-\frac{3}{3}+\frac{4}{3} x\right) \mathrm{H}_{0}+\left(\frac{4}{3}-\frac{4}{3} x\right) \mathrm{H}_{1}-\frac{38}{9} x+\frac{38}{9}\right\} \cdot \\
&\left(16 x+16-8 x^{2}+\frac{24}{5} x^{3}\right) \mathrm{H}_{0,0}+(8-8 x) \mathrm{H}_{1,0,0}+\left(8 x^{2}+12 x+12-\frac{24}{5} x^{3}\right) \zeta_{2}+ \\
&\left.(-8+8 x) \zeta_{3}+\frac{(x-1)\left(216 x^{2}+157 x+216\right)}{45 x}\right\}+
\end{aligned}
$$




\section{References}

[1] O. Biebel, P. Nason and B.R. Webber, (2001), hep-ph/0109282

[2] S. Kluth, (2006), hep-ex/0603011

[3] G. Curci, W. Furmanski and R. Petronzio, Nucl. Phys. B175 (1980) 27

[4] W. Furmanski and R. Petronzio, Phys. Lett. B97 (1980) 437

[5] E.G. Floratos, C. Kounnas and R. Lacaze, Nucl. Phys. B192 (1981) 417

[6] P. Nason and B.R. Webber, Nucl. Phys. B421 (1994) 473

[7] P.J. Rijken and W.L. van Neerven, Phys. Lett. B386 (1996) 422, hep-ph/9604436

[8] P.J. Rijken and W.L. van Neerven, Phys. Lett. B392 (1997) 207, hep-ph/9609379

[9] P.J. Rijken and W.L. van Neerven, Nucl. Phys. B487 (1997) 233, hep-ph/9609377

[10] A. Mitov, S. Moch and A. Vogt, (2006), hep-ph/0604053

[11] A. Mitov, (2005), hep-ph/0511340

[12] D.I. Kazakov and A.V. Kotikov, Nucl. Phys. B307 (1988) 721

[13] S. Moch and J.A.M. Vermaseren, Nucl. Phys. B573 (2000) 853, hep-ph/9912355

[14] S. Moch, J.A.M. Vermaseren and A. Vogt, Nucl. Phys. B688 (2004) 101, hep-ph/0403192

[15] A. Vogt, S. Moch and J.A.M. Vermaseren, Nucl. Phys. B691 (2004) 129, hep-ph/0404111

[16] J.A.M. Vermaseren, A. Vogt and S. Moch, Nucl. Phys. B724 (2005) 3, hep-ph/0504242

[17] S.A. Larin, T. van Ritbergen and J.A.M. Vermaseren, Nucl. Phys. B427 (1994) 41

[18] S.A. Larin et al., Nucl. Phys. B492 (1997) 338, hep-ph/9605317

[19] A. Retey and J.A.M. Vermaseren, Nucl. Phys. B604 (2001) 281, hep-ph/0007294

[20] J. Blümlein and J.A.M. Vermaseren, Phys. Lett. B606 (2005) 130, hep-ph/0411111

[21] S. Moch, (2005), math-ph/0509058

[22] V.N. Gribov and L.N. Lipatov, Sov. J. Nucl. Phys. 15 (1972) 438

[23] M. Stratmann and W. Vogelsang, Nucl. Phys. B496 (1997) 41, hep-ph/9612250

[24] D.J. Broadhurst and A.L. Kataev, Phys. Lett. B315 (1993) 179, hep-ph/9308274

[25] S.J. Brodsky et al., Phys. Lett. B372 (1996) 133, hep-ph/9512367

[26] J. Blümlein, V. Ravindran and W.L. van Neerven, Nucl. Phys. B586 (2000) 349, hep-ph/0004172

[27] L.N. Lipatov, Sov. J. Nucl. Phys. 20 (1975) 94

[28] Y.L. Dokshitzer, Sov. Phys. JETP 46 (1977) 641

[29] G. Altarelli and G. Parisi, Nucl. Phys. B126 (1977) 298

[30] S. Kretzer, Phys. Rev. D62 (2000) 054001, hep-ph/0003177

[31] L. Bourhis et al., Eur. Phys. J. C19 (2001) 89, hep-ph/0009101

[32] B.A. Kniehl, G. Kramer and B. Pötter, Nucl. Phys. B582 (2000) 514, hep-ph/0010289

[33] B.A. Kniehl, G. Kramer and B. Pötter, Nucl. Phys. B597 (2001) 337, hep-ph/0011155

[34] B. Mele and P. Nason, Nucl. Phys. B361 (1991) 626

[35] K. Melnikov and A. Mitov, Phys. Rev. D70 (2004) 034027, hep-ph/0404143 
[36] A. Mitov, Phys. Rev. D71 (2005) 054021, hep-ph/0410205

[37] G. 't Hooft and M. Veltman, Nucl. Phys. B44 (1972) 189

[38] C.G. Bollini and J.J. Giambiagi, Nuovo Cim. B12 (1972) 20

[39] J.F. Ashmore, Lett. Nuovo Cim. 4 (1972) 289

[40] G.M. Cicuta and E. Montaldi, Nuovo Cim. Lett. 4 (1972) 329

[41] W.A. Bardeen et al., Phys. Rev. D18 (1978) 3998

[42] P. Nason and C. Oleari, Phys. Lett. B447 (1999) 327, hep-ph/9811206

[43] P. Nason and C. Oleari, Nucl. Phys. B565 (2000) 245, hep-ph/9903541

[44] F.V. Tkachov, Phys. Lett. B100 (1981) 65

[45] K.G. Chetyrkin and F.V. Tkachov, Nucl. Phys. B192 (1981) 159

[46] F.V. Tkachov, Theor. Math. Phys. 56 (1983) 866

[47] C. Anastasiou and K. Melnikov, Nucl. Phys. B646 (2002) 220, hep-ph/0207004

[48] S. Moch, J.A.M. Vermaseren and A. Vogt, Nucl. Phys. B646 (2002) 181, hep-ph/0209100

[49] S.A. Larin, Phys. Lett. B303 (1993) 113, hep-ph/9302240

[50] S.A. Larin and J.A.M. Vermaseren, Phys. Lett. B259 (1991) 345

[51] S.G. Gorishnii, S.A. Larin and F.V. Tkachov, Phys. Lett. B124 (1983) 217

[52] S.G. Gorishnii and S.A. Larin, Nucl. Phys. B283 (1987) 452

[53] W.L. van Neerven, Nucl. Phys. B268 (1986) 453

[54] T. Matsuura, S.C. van der Marck and W.L. van Neerven, Nucl. Phys. B319 (1989) 570

[55] S. Moch, J.A.M. Vermaseren and A. Vogt, JHEP 08 (2005) 049, hep-ph/0507039

[56] C. Anastasiou and A. Lazopoulos, JHEP 07 (2004) 046, hep-ph/0404258

[57] T. van Ritbergen and R.G. Stuart, Nucl. Phys. B564 (2000) 343, hep-ph/9904240

[58] S. Laporta, Phys. Lett. B504 (2001) 188, hep-ph/0102032

[59] S. Laporta, Int. J. Mod. Phys. A15 (2000) 5087, hep-ph/0102033

[60] Y. Schröder, Nucl. Phys. Proc. Suppl. 116 (2003) 402, hep-ph/0211288

[61] J.A.M. Vermaseren, Int. J. Mod. Phys. A14 (1999) 2037, hep-ph/9806280

[62] S. Moch and P. Uwer, (2005), math-ph/0508008

[63] J.A.M. Vermaseren, (2000), math-ph/0010025

[64] A. Gehrmann-De Ridder, T. Gehrmann and G. Heinrich, Nucl. Phys. B682 (2004) 265, hep-ph/0311276

[65] D.I. Kazakov, Phys. Lett. B133 (1983) 406

[66] R.K. Ellis, W.J. Stirling and B.R. Webber, $Q C D$ and collider physics Cambridge Monogr. on Part. Phys., Nucl. Phys. and Cosmol. 8 (1996)

[67] E. Remiddi and J.A.M. Vermaseren, Int. J. Mod. Phys. A15 (2000) 725, hep-ph/9905237

[68] D.A. Kosower and P. Uwer, Nucl. Phys. B674 (2003) 365, hep-ph/0307031

[69] T. Gehrmann, T. Huber and D. Maitre, Phys. Lett. B622 (2005) 295, hep-ph/0507061

[70] J. Blümlein and V. Ravindran, (2006), hep-ph/0604019 